\title{
Near infrared photometry of IRAS sources with colours like planetary nebulae. III. ${ }^{\star, \star \star}$
}

\author{
P. García-Lario ${ }^{1}$, A. Manchado ${ }^{2}$, W. Pych ${ }^{3}$, and S.R. Pottasch ${ }^{4}$ \\ 1 Leiden Observatory. PO Box 9513, NL-2300 RA Leiden, The Netherlands \\ 2 Instituto de Astrofísica de Canarias, E-38200 La Laguna, Tenerife, Spain \\ 3 Warsaw University Astronomical Observatory, Al. Ujazdowskie 4, 00-478 Warszawa, Poland \\ ${ }^{4}$ Kapteyn Laboratorium, PO Box 800, NL-9700, AV Groningen, The Netherlands
}

Received July 23, 1996; accepted April 7, 1997

\begin{abstract}
We present the near infrared photometry of a new sample of 225 IRAS sources, many of them previously unidentified in the literature, selected because their far infrared colours are similar to those shown by known planetary nebulae. The results obtained are used to establish the main source of near infrared emission. Combining this information with the far infrared IRAS data and a few additional criteria we determine the nature and evolutionary stage of all the sources observed so far, including those for which near infrared photometry was previously reported in Papers I and II.
\end{abstract}

Among the unidentified IRAS sources in our sample we find only a small percentage of planetary nebulae, many of them very young and dusty, showing peculiar near infrared colours. Most of the new objects observed in the near infrared are identified as transition objects in the previous stages of the stellar evolution. Among them, we find heavily obscured late-AGB stars, early post-AGB stars still obscured by thick circumstellar envelopes which are probably the true progenitors of planetary nebulae, and a significant fraction of stars with bright optical counterparts showing little or no near infrared excess, which we associate with highly evolved post-AGB stars with low mass progenitors, which may never become planetary nebulae. In addition, we also find a small percentage of young stellar objects, as well as a few Seyfert galaxies.

We conclude that, in most cases, based on near infrared data alone, it is not possible to give a confident classification of the unidentified IRAS source. However, the near infrared is shown to be a powerful tool, specially when dealing with objects which are heavily obscured in

Send offprint requests to: P. García-Lario

* Based on observations collected at the European Southern Observatory, La Silla (Chile) and at the Spanish Observatorio del Teide, Tenerife, Spain.

** Table 6 is only available electronically at the CDS via anonymous ftp to cdsarc.u-strasbg.fr (130.79.128.5) or via http: //cdsweb.u-strasbg.fr/Abstract.html the optical. In this case, the detection of the near infrared counterpart is the only way in which we can extend the study of these sources to other spectral ranges and may be crucial to understand the short-lived phase which precedes the formation of a new planetary nebula.

Key words: stars: AGB and Post-AGB - infrared: general - planetary nebulae: general

\section{Introduction}

This is the third Paper of a series containing near infrared photometry of a sample of IRAS sources with far infrared colours similar to those shown by planetary nebulae (PNe, hereafter). In the previous ones, Manchado et al. (1989a; Paper I) and García-Lario et al. (1990; Paper II) showed preliminary results obtained on a subsample of these IRAS sources. The selection criteria were previously described in Papers I and II. They are based on the fact that $\mathrm{PNe}$ and objects in the transition phase from the AGB to the PN stage are strong infrared emitters with peculiar infrared colours. Thus, it is possible to separate a region in the IRAS two-colour diagram $12 \mu \mathrm{m} / 25 \mu \mathrm{m}$ vs. $25 \mu \mathrm{m} / 60 \mu \mathrm{m}$ where most of the objects found are PNe or objects connected with this evolutionary stage. Only a small overlap exists with other objects, like Seyfert galaxies, compact H II regions and T-Tauri stars (Pottasch et al. 1988). The complete sample satisfying our selection criteria contains 1084 sources (García-Lario 1992), all them well detected in at least the 12,25 and $60 \mu \mathrm{m}$ bands. About $50 \%$ of them are previously unidentified objects or poorly known in the literature. Among the well identified ones, we find a majority of $\mathrm{PNe}(49 \%)$ and other post-main sequence stars ( 27 small fraction of young stellar objects (17\%) and active galactic nuclei $(7 \%)$. 
Table 1. Log-in of the observations

\begin{tabular}{lcrc}
\hline & Period & Telescope & $\begin{array}{c}\text { Observed(detected) } \\
\text { IRAS sources }\end{array}$ \\
\hline & & & \\
$(1)$ & May 23, 1989 - June 5, 1989 & $1.5 \mathrm{~m} \mathrm{CST}$ & $28(26)$ \\
$(2)$ & November 26, 1989 - December 4, 1989 & $1.5 \mathrm{~m} \mathrm{CST}$ & $6(6)$ \\
$(3)$ & April 18, 1990 - April 24, 1990 & $1.5 \mathrm{~m} \mathrm{CST}$ & $19(17)$ \\
$(4)$ & May 6, 1990 - May 11, 1990 & $1 \mathrm{~m} \mathrm{ESO}$ & $64(56)$ \\
$(5)$ & June 19, 1990 - June 25, 1990 & $1.5 \mathrm{~m} \mathrm{CST}$ & $65(48)$ \\
$(6)$ & March 19, 1992 - March 25, 1992 & $1 \mathrm{~m}$ ESO & $31(29)$ \\
$(7)$ & May 16, 1992 - May 22, 1992 & $1 \mathrm{~m} \mathrm{ESO}$ & $23(21)$ \\
$(8)$ & October 15, 1992 - October 21, 1992 & $1.5 \mathrm{~m} \mathrm{CST}$ & $10(9)$ \\
$(9)$ & December 1, 1993 - December 7, 1993 & $1.5 \mathrm{~m} \mathrm{CST}$ & $52(42)$ \\
& & & \\
\hline
\end{tabular}

The number of objects known in the short transition phase which precedes the formation of a $\mathrm{PN}$ is very small. The final aim of this work is to increase this number through the detection of new candidates among the infrared sources included in the IRAS Point Source Catalogue (PSC) with no previous identification and the adequate colours. Many of them are expected to be heavily obscured by the thick circumstellar envelopes formed during the AGB phase. The determination of the near infrared counterpart is, thus, the natural extension toward bluer wavelengths in the study of these sources and essential for subsequent studies in this or other spectral ranges.

The near infrared photometry, as we proved in Papers I and II, can be used to determine whether the main origin of the emission observed is stellar, nebular or due to the dust present in the circumstellar envelope. According to this, we can try to identify the nature and evolutionary stage of the sources observed since each type of object in the sample shows characteristic near infrared properties which can be used to recognize them. Many PNe, for instance, are known to exhibit a characteristic $J$ band excess due to the presence of an emission line of He I at $1.083 \mu \mathrm{m}$ (Whitelock 1985). Also very late-AGB stars and heavily obscured post-AGB stars can be easily recognized because of their extremely reddened near infrared colours (Le Bertre 1988; van der Veen et al. 1989). In many cases, however, the identification of a given source is not possible based on near infrared data alone. Compact H II regions, T-Tauri stars and active galaxies which, as we have refered above, are also present in our sample, sometimes show near infrared colours very similar to those observed in lateAGB and post-AGB stars. In this case, the combination with information obtained in other spectral ranges or the use of additional criteria is needed. Sometimes the problem is easy to solve, as for OH/IR stars, which are charac- terized by the presence of a double-peaked $\mathrm{OH}$ maser emission at $1612 \mathrm{MHz}$. Unfortunately, this emission is usually not observed in more evolved stars. In addition, optically bright post-AGB stars show no or very little near infrared excess and, thus, are very difficult to distinguish from foreground sources. Confusion is a major problem specially when observing towards the galactic bulge, where fields are frequently crowded.

Apart from a few exceptions, observations have been made only for sources satisfying our selection criteria with no previous near infrared measurements. They are described in Sect. 2. In Sect. 3.1 we analyse the near infrared properties of the various types of stellar objects found among very well identified IRAS sources included in our sample for which data are available in this spectral range in the literature. These data will be used for comparison in our analysis of the unidentified objects. The same kind of analysis is done with their far infrared emission in Sect. 3.2, as derived from IRAS data. The results obtained are shown in Sect. 4. Combining the characteristics of the near infrared emission with the IRAS properties and a few additional criteria we classify all the sources observed so far, including those for which the near infrared photometry was previously reported in Papers I and II. The conclusions derived are presented in Sect. 5 .

This work is part of a wide multiwavelength survey which combines infrared data with optical spectroscopy (whenever an optical counterpart is found) and radio continuum observations. The analysis of the results obtained in other wavelength ranges will be published elsewhere (van de Steene et al. 1996a,b; García-Lario et al. 1997a). Optical and near infrared finding charts together with the accurate coordinates of the counterparts found will be presented in forthcoming papers. 


\section{Observations}

The results presented here include the near infrared photometry carried out in the standard $J(1.25 \mu \mathrm{m})$, $H \quad(1.65 \mu \mathrm{m}), \quad K(2.2 \mu \mathrm{m})$, and in some cases, $L^{\prime}(3.8 \mu \mathrm{m})$ and $M(4.8 \mu \mathrm{m})$ bands during different observing runs both with the $1.55 \mathrm{~m}$ CST telescope operated by the Instituto de Astrofísica de Canarias at the Spanish Observatorio del Teide (Tenerife, Spain), and with the $1 \mathrm{~m}$ ESO telescope at the Observatorio de La Silla (Chile) since May 1989 until December 1993. The log-in of the observations is shown in Table 1, where we also quote the number of objects observed during each run, together with the number of objects detected (in brackets) in each case.

At both telescopes we used infrared photometers equipped with InSb photovoltaic detectors, operating at the temperature of liquid nitrogen, with a photometric aperture of $15^{\prime \prime}$ and a chopper throw of $20^{\prime \prime}$ in R.A. direction. The Teide photometric system is described in Arribas \& Martínez-Roger (1987), as well as its relations with other standard photometric systems. The ESO photometric system is described in Bouchet et al. (1991). For flux calibration we used the list of standard stars given by Koornneef (1983) in the case of those stars observed at the CST while, while for those observed at the $1 \mathrm{~m} \mathrm{ESO}$ telescope we used the standard stars included in Bouchet et al. (1991). Several standard stars were observed at least twice each night at different air masses to determine the atmospheric extinction in each filter.

Prior to our observations, we searched the ESO or Palomar blue and red prints looking for the presence of possible optical counterparts. Usually only one candidate was found inside the IRAS error ellipse but sometimes, specially towards the Galactic Center, several objects were observed and, in other cases, nothing was seen around the IRAS position. When several possible optical counterparts are found in a goven field, a good method to determine the which one is the best candidate is to compare the blue and red prints, since one should expect these stars to be strongly reddened as a consequence of the dust present in their circumstellar envelopes. Unfortunately, as we have already mentioned, this is not always valid. If no optical counterpart is observed, we chose a reference star nearby, bright enough to be detected on the TV screen at the telescope, and calculate the blind offset necessary to move the telescope to the IRAS position.

Once at the telescope we made raster scans $30^{\prime \prime} \times 30^{\prime \prime}$ wide centered on the IRAS position in the $K$ band resulting in an $80 \%$ of positive detections. The limiting magnitude is estimated to be between 11th and 13th with this method at both telescopes depending on the atmospheric conditions. Usually a single bright near infrared counterpart was found in each field, in most cases coincident with the best candidate previously determined through the visual inspection of the ESO or Palomar prints. If more than one near infrared source was detected, we always measured that closest to the original IRAS coordinates. Mean discrepancies between the IRAS coordinates and the position of the near infrared counterparts found are around $16^{\prime \prime}$ in right ascension and $8^{\prime \prime}$ in declination. In a few cases, however, our identification is more doubtful, since the near infrared counterpart was found at distances of around $1^{\prime}$ from the IRAS position. In these cases, one must take into consideration other circumstances such as, for instance, the characteristics of the optical spectrum, if available, or whether the near infrared and optical brightness are consistent with the properties observed in other spectral ranges, as we will discuss later. It is important to remark that, although we cannot rule out the possibility of having observed spurious sources in a few cases, the probability of this is very small and we are confident that this does not affect the statistical conclusions derived in this Paper.

In the absence of problems, and once the near infrared counterpart was determined, we performed the photometry in the standard $J, H$ and $K$ filters. $L^{\prime}$ and $M$ were used only in the case of very good atmospheric conditions (humidity below 40\%) and when the object was bright enough (or red enough) to expect a positive detection in these two filters.

\section{Analysis of the infrared properties of well identified sources in the sample}

\subsection{Near infrared emission}

For a better understanding of the results shown in this Paper it is worth to study first, making use of the data available in the literature (Gezari et al. 1993), the characteristics of the near infrared emission observed in the different type of objects which are expected to be present in our sample. As refered above, among them, we can find not only $\mathrm{PNe}$ and other post-main sequence stars evolutionary connected with $\mathrm{PNe}$, such as late-AGB and postAGB stars, but also, although in a small proportion, a wide variety of young stellar objects, like T-Tauri stars, Herbig Ae/Be stars or compact H II regions, and a few active galaxies.

For an adequate interpretation of the data we need to consider the different sources of near infrared flux which can contribute to the observed emission:

a) Thermal emission from plasma: basically recombination continuum and free-free emission from nebular hydrogen and helium, only expected when ionization is present.

b) Thermal emission from dust: the emission due to the cold dust present in circumstellar envelopes at characteristic temperatures lower than $300 \mathrm{~K}$ is not expected to appreciably contribute to the emission observed in the near infrared. However, the presence of hot dust $(T \sim 1000 \mathrm{~K})$ can easily be detected in the near infrared, specially in the reddest bands, and may be interpreted as a consequence 


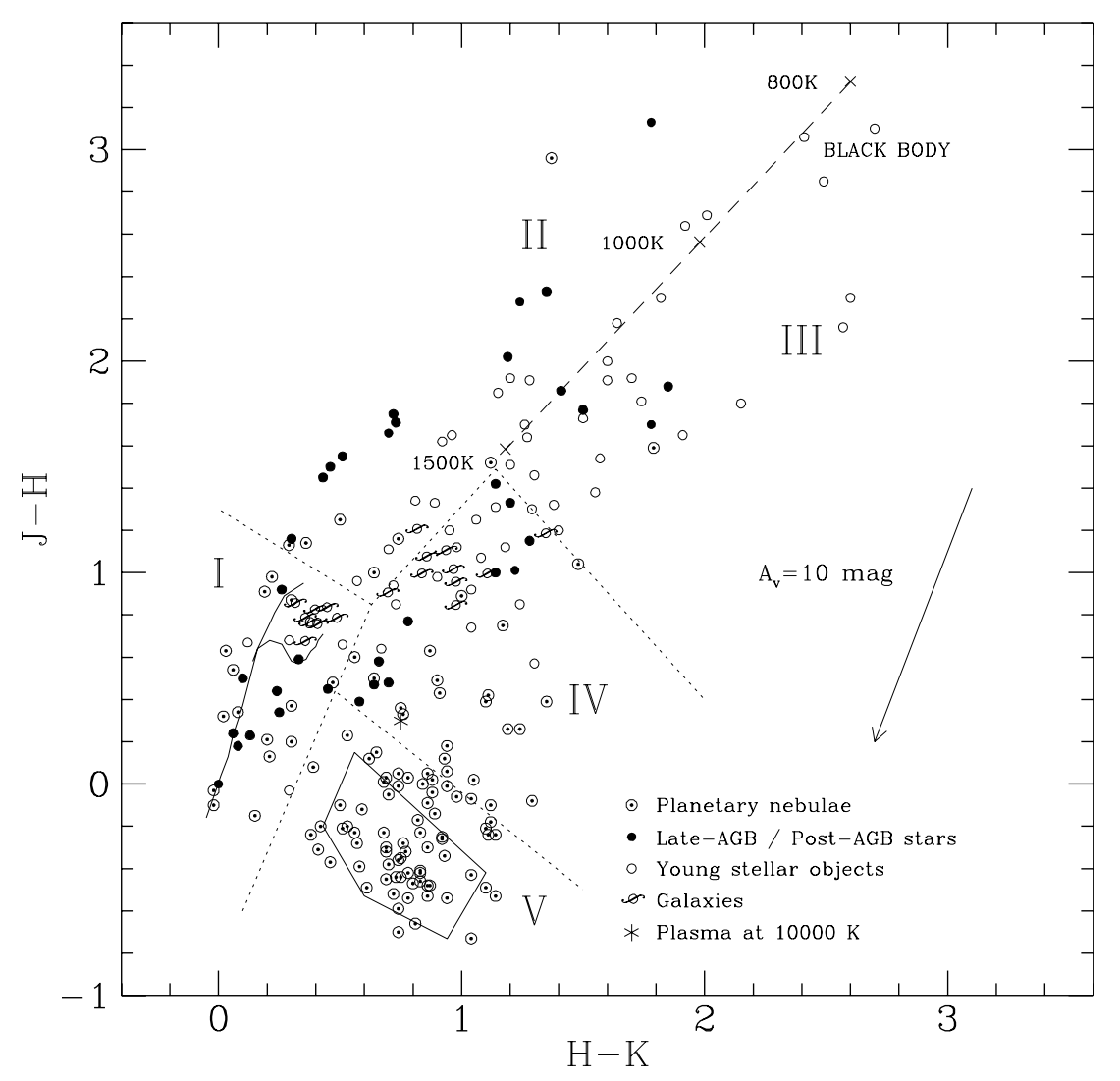

Fig. 1. $J-H$ vs. $H-K$ two-colour diagram where we show the position of very well identified objects present in our sample for which data are available in the literature. The solid line in Region I indicates the position corresponding to main sequence and giant stars, for comparison (see text for more details)

of recent mass loss or, in the case of young stellar objects, as an indicator of the presence of a circumstellar disk.

c) Stellar continuum: it can dominate only in the case of stars not heavily obscured by the circumstellar dust. The hot central stars of PNe emit basically in the ultraviolet, and thus, must be extremely bright to detect its Rayleigh-Jeans tail emission in the near infrared.

d) Emission lines: basically recombination lines from hydrogen and helium. They can be the main source of near infrared emission in the range between 1 and $2 \mu \mathrm{m}$ for ionized envelopes. The neutral helium triplet at $1.083 \mu \mathrm{m}$ can sometimes be bright enough to completely dominate the emission in the $J$ band, even although this emission feature is located close to the edge of the photometric band. In addition, the Paschen recombination lines of hydrogen can also contribute to the $J$ emission, while Brackett and Pfund lines and, in some cases, molecular hydrogen emission would affect the emission observed at the $H$ and $K$ bands.

In Fig. 1 we show a near infrared two-colour diagram $(J-H$ vs. $H-K)$ where we have plotted together the posi- tion of the different types of objects present in our sample for which data are available in the literature. We have divided this diagram into several regions (from I to V), which will be used in our subsequent analysis. The colours are not extinction corrected, since the value of the extinction is, in most cases, not known or bad determined. As an indication, a vector representing the effect of reddening is included in this diagram, together with the positions corresponding to main-sequence and giant stars, black-body emission at different temperatures and that corresponding to a plasma at a temperature of $10^{4} \mathrm{~K}$ (Whitelock 1985).

The distribution of the various types of objects found in the near infrared two-colour diagram is presented in Table 2. In the following we will try to characterize each class of object according to their near infrared properties.

\subsection{1. $\mathrm{PNe}$}

PNe have only recently been systematically observed in the near infrared. Before 1985 only a few $\mathrm{PNe}$, the brightest ones, had been observed in this wavelength range (Willner et al. 1972; Allen 1973; Persson \& Frogel 
Table 2. Distribution of the various types of well identified IRAS sources in our sample in the near infrared two-colour diagram (data taken from the literature)

\begin{tabular}{lcccccc}
\hline Class & Region I & Region II & Region III & Region IV & Region V & Total \\
\hline Planetary Nebulae & 14 & 7 & 1 & 22 & 69 & 113 \\
Late-AGB/Post-AGB stars & 9 & 12 & 3 & 10 & 0 & 34 \\
Young Stellar Objects & 4 & 13 & 21 & 14 & 0 & 52 \\
Galaxies & 8 & 1 & 0 & 9 & 0 & 18 \\
& & & & & & \\
\hline
\end{tabular}

1973; Allen \& Glass 1974). More recently, several surveys have been carried out providing near infrared photometry for more than $200 \mathrm{PNe}$ in the $J, H$ and $K$ bands and, in some cases, also in $L^{\prime}$ and $M$ (Whitelock 1985; Kwok et al. 1986; Peña \& Torres-Peimbert 1987; Persi et al. 1987). Most of these PNe satisfy our selection criteria and are, therefore, included in our sample. In Fig. 1 we show their position in the near infrared twocolour diagram $J-H$ vs. $H-K$.

From the analysis of Fig. 1 it is clear that there is a strong concentration of sources in Region $\mathrm{V}$, specially around the so-called nebulae box, as defined by Whitelock (1985), which has also been represented in this figure with a solid line. Around two thirds of the PNe observed in the near infrared fall inside or in the surroundings of this box. This confined region of the two-colour diagram is well separated from that where main-sequence and giant stars are located and shows no overlap with any other type of stellar object. PNe in Region $\mathrm{V}$ show a characteristic $J$ band excess with respect to the emission expected from a plasma at an electronic temperature of $10^{4} \mathrm{~K}$. This effect cannot be attributed to a different $T_{\mathrm{e}}$ in the plasma, since this would only produce a small displacement up and left (if $T_{\mathrm{e}}$ increases) or down and right (if $T_{\mathrm{e}}$ decreases) in the diagram, but it is probably due to the presence of the strong He I triplet at $1.083 \mu \mathrm{m}$.

This hypothesis was confirmed by Whitelock (1985) and Peña \& Torres-Peimbert (1987), who found a clear correlation between the $J-H$ extinction corrected infrared colour and the $\mathrm{He}^{+}$abundance. In fact, the emission excess in the $J$ band is more pronounced in intermediate excitation class PNe. In very high excitation PNe the He I triplet is very weak, although the He II emission line at $1.162 \mu \mathrm{m}$ can still contribute to a small $J$ excess. On the other hand, in very low excitation PNe, most of the helium is in neutral state, producing a much weaker He I triplet and, thus, a smaller $J$ excess.

A considerable number of $\mathrm{PNe}$ lie close to the borderline between Regions IV and V above and to the right of the nebulae box, and a few of them can also be found in Region III, as we show in Fig. 1. Although this anomalous location could simply be the result of interstellar reddening, it can also be explained as the effect of hot dust present in the circumstellar envelope. This would produce a characteristic excess in the longer wavelengths and, thus, a larger value of the $H-K$ colour index. The presence of hot dust at temperatures around $1000 \mathrm{~K}$ in most of these $\mathrm{PNe}$ is confirmed by the fact that they also show large values of $K-L^{\prime}$ which cannot be explained by the effect of interstellar reddening. Remarkably, most of these dusttype $\mathrm{PNe}$ are considered to belong to the youngest group of PNe, such as M2-9, Vy2-2, IC 418, Hu2-1, Sw St1, K3-62, IC 5117 or Tc 1 . We interpret the presence of hot dust in their envelopes as the result of mass loss processes suffered in the very recent past or even still taking place, as the P-Cygni profiles found in some cases in their spectra indicate.

Finally, a small number of $\mathrm{PNe}$ show near infrared colours similar to those of main sequence stars and giants. Probably the central stars of these stellar-type PNe are binary systems and we are only detecting the emission coming from the companion star in the near infrared. Another possibility is the presence of a foreground source in the aperture which is contaminating the near infrared photometry. This kind of near infrared emission is observed, for instance, in $\mathrm{He} 2-138$, IC 3568, Me2-2, NGC 5315, and $\mathrm{Pb} 8$, all them located in Region I of our diagram. Clearly, additional observations are needed for all them.

The peculiar location of a few PNe in Region II deserves a special attention. Most of them, such as M3-39 or H2-1, are close to Region I, and the near infrared emission observed can be explained as the combination of a moderate infrared excess with a stellar-type emission. The most extremely reddened colours observed are those of M1-26, whose optical spectrum corresponds to a very low excitation $\mathrm{PN}$ with its central star showing an effective temperature of only $25000 \mathrm{~K}$ (Kwok 1985). Its location in the HR diagram (West \& Kohoutek 1985) and the optical 
and radio compact appearance indicates that $\mathrm{M} 1-26$ is a very young $\mathrm{PN}$, for which the circumstellar extinction is still very high. Very similar characteristics are observed in the very well known proto-PN CRL 618 (Latter et al. 1992).

\subsubsection{Late-AGB/Post-AGB stars}

While still on the AGB, stars are strongly variable, due to stellar pulsation. They are then called "variable $\mathrm{OH} / \mathrm{IR}$ stars", since most of them show a double-peaked $\mathrm{OH}$ maser emission at $1612 \mathrm{MHz}$ and are very bright in the infrared, heavily obscured by thick circumstellar envelopes formed as a consequence of the strong mass loss. Shortly after the end of the AGB, the mass loss suddenly stops and for no longer the star is variable, while the effective temperature of the central star increases. The star is now in the post-AGB phase and recognized as a "nonvariable $\mathrm{OH} / \mathrm{IR}$ star" while the $\mathrm{OH}$ maser emission is still detectable. After the partial dilution of the circumstellar envelope in the interstellar medium the $\mathrm{OH}$ maser emission dissapears and the central post-AGB star becomes observable again in the optical, in its way to become a new PN.

In Fig. 1 we can see that IRAS sources recently identified as late-AGB or post-AGB stars in the literature show a wide distribution in the near infrared two-colour diagram and can be located everywhere, with the only exception of Region V. Fortunately, objects in different regions of the diagram show peculiar characteristics which can help us in our identification purposes. For instance, most of the strongly reddened objects found in Region II are identified as $\mathrm{OH} / \mathrm{IR}$ stars since they show $\mathrm{OH}$ maser emission and no optical counterparts. Variable OH/IR stars in this region of the diagram show near infrared properties which are an extension towards more extreme values of those observed in optically bright Mira variables, which are also stars in the AGB. Mira variables are not plotted in our diagram because they do not fulfill our selection criteria, but many of them are also known to be located in Region II, inmediately above but close to Region I (Feast \& Whitelock 1987). Objects in Regions III and IV, on the other hand, are also affected by a strong circumstellar reddening but their slightly different position can simply be due to an excess of emission in the $K$ band produced by the presence of hot dust in the envelope. Some of them are not variable in the near infrared and may be identified as early post-AGB stars. While objects in Region III usually show $\mathrm{OH}$ maser emission and no optical counterparts, those in Region IV are not so frequently detected in the $\mathrm{OH}$ maser line. Moreover, they are not so strongly obscured and sometimes show a faint optical counterpart. Finally, the objects found in Region I, showing a stellarlike emission with little or no reddening, are identified as evolved post-AGB stars, now observable again in the optical after the dilution of the circumstellar envelope in the interstellar medium. They show a small irregular variability and no $\mathrm{OH}$ maser emission. Some near infrared excess is observed in a few of them located to the right of the main-sequence. This is probably indicative of recent post-AGB mass-loss, something which, for some of these objects, has been confirmed through the detection of $\mathrm{H} \alpha$ emission in the optical spectrum.

\subsubsection{Young stellar objects}

Under this category we can find both heavily obscured young stellar objects showing the most extremely reddened colours, together with optically bright stars with little or just a moderate near infrared excess.

Among the first group it is possible to identify deeply obscured compact HII regions and Herbig-Haro objects still embedded in the molecular clouds in which they have been originated. They are predominantly located in Region III of the near infrared two-colour diagram, although a few are also found in Region II, always close to the position expected for black-bodies emitting at temperatures between 800 and $1500 \mathrm{~K}$.

The second group is basically formed by T-Tauri and Herbig Ae/Be stars, which are not so heavily obscured. Most of them are located in Region IV, although we also detect a few in Regions I and II. The circumstellar disks usually associated to these objects are probably the responsible for the presence of the near infrared excess observed (Strom et al. 1989; Hillenbrand et al. 1992). Finally, it is important to remark that, again, none of these objects is found in Region $\mathrm{V}$ of the diagram.

\subsubsection{Galaxies}

The small number of galaxies found satisfying our selection criteria are known to show active nuclei and most of them are classified in the literature as bright Seyfert galaxies. Although we expect to find a very small number of them among the unidentified objects in our sample it is worth to investigate whether they show peculiar near infrared colours which could be used for their identification.

As we can see in Fig. 1, they are all well confined in a relatively small region of the diagram in the intersection of Regions I and IV. Unfortunately, this is the same location in which we can also find, as we have already mentioned, evolved stars, young stellar objects and, sometimes, even PNe.

Seyfert galaxies detected by IRAS have previously been studied in this wavelength range by different authors (Sanders et al. 1988; Carico et al. 1990). They have shown that those with the higher luminosities usually show a near infrared excess originated in the circumnuclear regions, probably associated to a strong star-forming activity. According to this, we suggest that the group of galaxies in our sample with a moderate near infrared excess, located in Region IV, must possess starburst nuclei. 


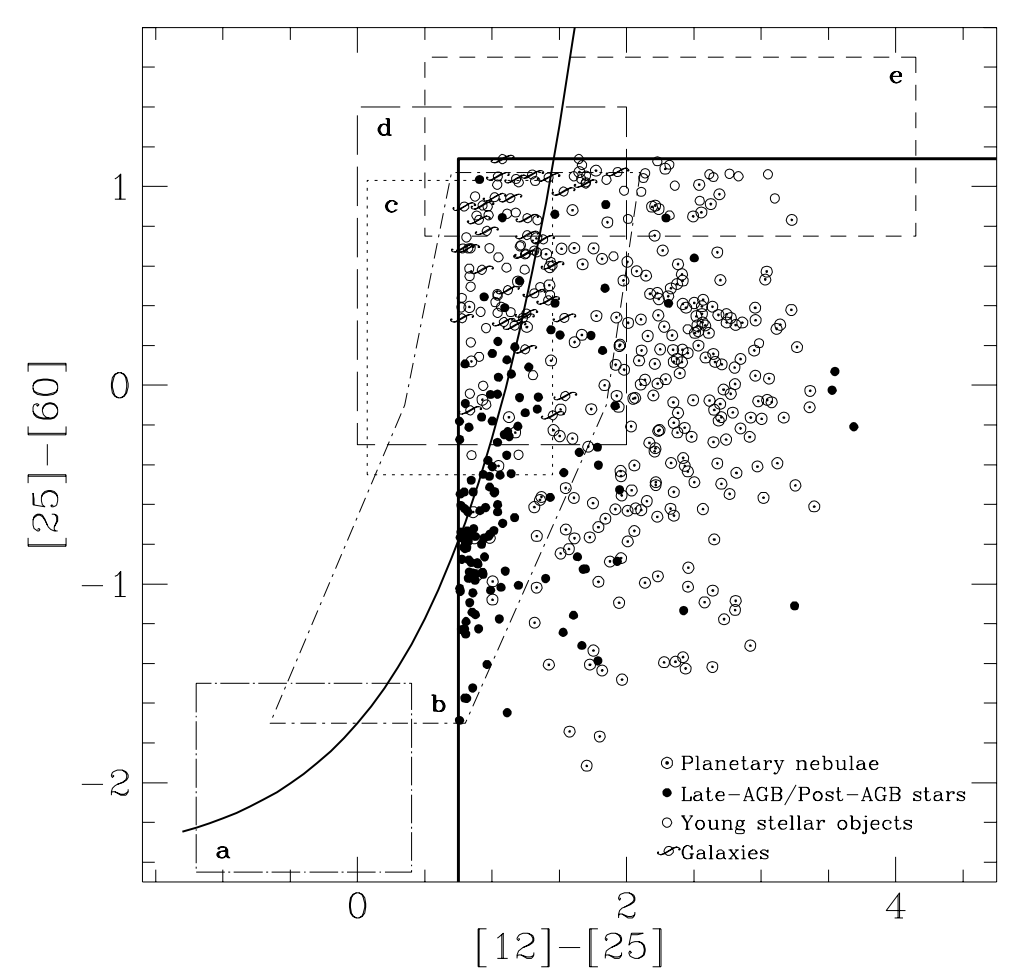

Fig. 2. IRAS two-colour diagram where we show the position of very well identified objects in our sample together with the regions associated to a) Optically bright Mira variables; b) Variable OH/IR stars; c) T-Tauri and Herbig Ae/Be stars; d) Active galactic nuclei; and e) Compact HII regions. The thick solid line indicates the limits used for the selection of our sample and the exponential curve represents the evolutionary track followed by AGB stars with increasing mass loss (see text for details)

On the other hand, the galaxies found in Region I of our diagram, showing stellar-like colours, may correspond to those with just a moderate nuclear activity, in which the dominant emission observed is originated in the outer disk and is basically due to the stellar content of the galaxy.

\subsection{Far infrared emission}

From the results above shown it is clear that, in most cases, it is not possible to determine, based on near infrared data alone, the nature of previously unidentified IRAS sources in our sample. Thus, additional criteria are needed to be used in combination with the near infrared photometry. Unfortunately, the only information available for many of the sources observed comes from IRAS data, since no observations in other spectral ranges are yet available.

In order to investigate whether a more detailed study of the far infrared properties shown by the variety of objects found in our sample could be used to provide useful colour classification criteria, we have plotted in Fig. 2 an IRAS two-colour diagram $[12]-[25]$ vs. $[25]-[60]$, where

$$
\begin{aligned}
& {[12]-[25]=-2.5 \log \frac{F_{12 \mu \mathrm{m}}}{F_{25 \mu \mathrm{m}}}} \\
& {[25]-[60]=-2.5 \log \frac{F_{25 \mu \mathrm{m}}}{F_{60 \mu \mathrm{m}}} .}
\end{aligned}
$$

In this plot we show the region of the diagram satisfying our selection criteria which, as we know, basically corresponds to that where most of the well known PNe are located (Pottasch et al. 1988), together with those associated to: a) Optically bright Mira variables (Herman 1988) b) Variable OH/IR stars (Sivagnaman 1989; te Lintel Hekkert et al. 1991); c) T-Tauri and Herbig Ae/Be stars (Harris et al. 1988); d) Active galactic nuclei (de Grijp et al. 1987; Kailey \& Lebofsky 1988); and e) Compact HII regions (Antonopoulos \& Pottasch 1987). The exponential curve crossing the diagram corresponds to the sequence of colours expected from model 
predictions for stars losing mass at increasing rate at the end of the AGB (Bedijn 1987).

As we can see, although a strong overlap exists between different kind of objects in specific areas of the diagram, it is also possible to identify wide regions in it where this overlap is minimum or restricted to only two different groups. The consistency of this colour association have been checked by plotting in Fig. 2 the position of all the well identified sources in our sample. Galaxies and young stellar objects show characteristic far infrared colours in excellent agreement with the predictions, and appear concentrated in specific areas of the diagram, while $\mathrm{PNe}$ are widely distributed, as already known. On the other hand, most of the objects classified as late-AGB/post-AGB stars in the diagram are concentrated in the region associated with variable $\mathrm{OH} / \mathrm{IR}$ stars, as expected. Most of them are known to show variable $\mathrm{OH}$ maser emission. The few objects in this class located well outside the limits of this Region are either heavily obscured non-variable $\mathrm{OH} / \mathrm{IR}$ stars, already in the post-AGB stage, or optically bright post-AGB stars with a supergiant-like spectrum, as we will see in the following section.

Apart from the IRAS photometry, mid-infrared IRAS Low Resolution Spectra (LRS) have also been used in our identification process, although they are only available for the brightest sources in our sample (Olnon \& Raimond 1986). LRS spectra are classified according to the slope of the continuum and the presence or absence of specific features in the spectrum (see IRAS Explanatory Supplement 1985). The LRS classes $3 n$ and $7 n$, for instance, correspond to objects with a very red continuum and a strong silicate absorption feature around $9.8 \mu \mathrm{m}$, where $n$ is a number from 1 to 9 increasing with the strength of this absorption. Well identified heavily obscured $\mathrm{OH} / \mathrm{IR}$ stars in our sample are allways associated with one of these two LRS classes. On the other hand, the LRS class $9 n$ is characteristic of evolved $\mathrm{PNe}$, since it corresponds to emission line spectra, where $n$, in this case, is a number from 1 to 6 increasing with the excitation class. Unfortunately, very few young stellar objects and galaxies are bright enough to have an available LRS. When this is the case, the LRS class $5 n$, which corresponds to featureless spectra with a red continuum, is frequently observed.

Finally, we have also used the IRAS variability index as an additional source of information for the classification of the unidentified objects in our sample. This variability index is a number between 0 and 99 which indicates the likelihood of variability for a given IRAS source. It is based on the fact that the inclusion of an infrared source in the Point Source Catalogue required its detection in at least two different scans, which could be separated by hours, weeks or months. In this way it is possible to have some information about the variability of the source. Moreover, the way in which this index was computed favours the association of the highest values with long-period variables. Well known variable $\mathrm{OH} / \mathrm{IR}$ stars showing smooth long- term variations are usually found associated to values well above $50 \%$, with a strong concentration around $99 \%$, while other variable objects in our sample, such as T-Tauri stars, with small amplitudes and irregular variations are associated to lower values of the variability index (below 50\%). It is important to remark that a low variability index associated to an $\mathrm{OH} / \mathrm{IR}$ star does not necessarily mean that the source is actually non-variable. Objects with very long periods may look like non-variable if they were detected in scans separated just a few weeks and there are ecliptic positions which were poorly scanned by IRAS where variable sources were missed (Whitelock et al. 1994). On the other hand, variable $\mathrm{OH} / \mathrm{IR}$ stars may also be misidentified as non-variable when the observations were taken at different epochs corresponding to a similar phase in the light curve. In contrast, a high variability index can only be associated to true variable stars.

\section{Results}

\subsection{Near infrared photometry of the unidentified IRAS sources in the sample}

In Tables $3 \mathrm{a}$ to $\mathrm{g}$ we show the photometric magnitudes of the 225 IRAS sources measured in the near infrared, together with the estimated associated errors and a number indicating the run in which the source was observed, according to the list given in Table 1. Letters A and B, following the IRAS name, indicate that two near infrared counterparts were found equidistant to the nominal IRAS position. In addition, in Table 4 , we list the IRAS names of the 42 sources not detected in the $K$ band. Although in many cases they are associated with the faintest sources in our sample showing the lower fluxes at $12 \mu \mathrm{m}$, some of them were detected at a different epoch with a $K$ magnitude well above the detection limit, which may indicate a strong variability. As we can see, there is a high percentage of positive detections, around $80 \%$, which confirms the validity of the method of observation used.

All the IRAS sources included in Table 3 satisfy the selection criteria described in Paper I with the exceptions of IRAS $19344+2457$, identified as a new OH/IR star, and for which no previous near infrared photometry was available, and IRAS 19590-1249, a recently discovered hot post-AGB star at high galactic latitude with nebular emission lines (McCausland et al. 1992). Both show far infrared colours very similar to those required to be included in the sample but do not strictly satisfy all the selection criteria.

We have plotted in Fig. 3 the position of the observed infrared sources in the near infrared two-colour diagram $J-H$ vs. $H-K$. Again, as in Fig. 1, we have divided the diagram into Regions (from I to V) for our analysis.

The distribution observed in Fig. 3 looks quite different to that shown by the sample of well identified objects in Fig. 1, specially the fact that only three 


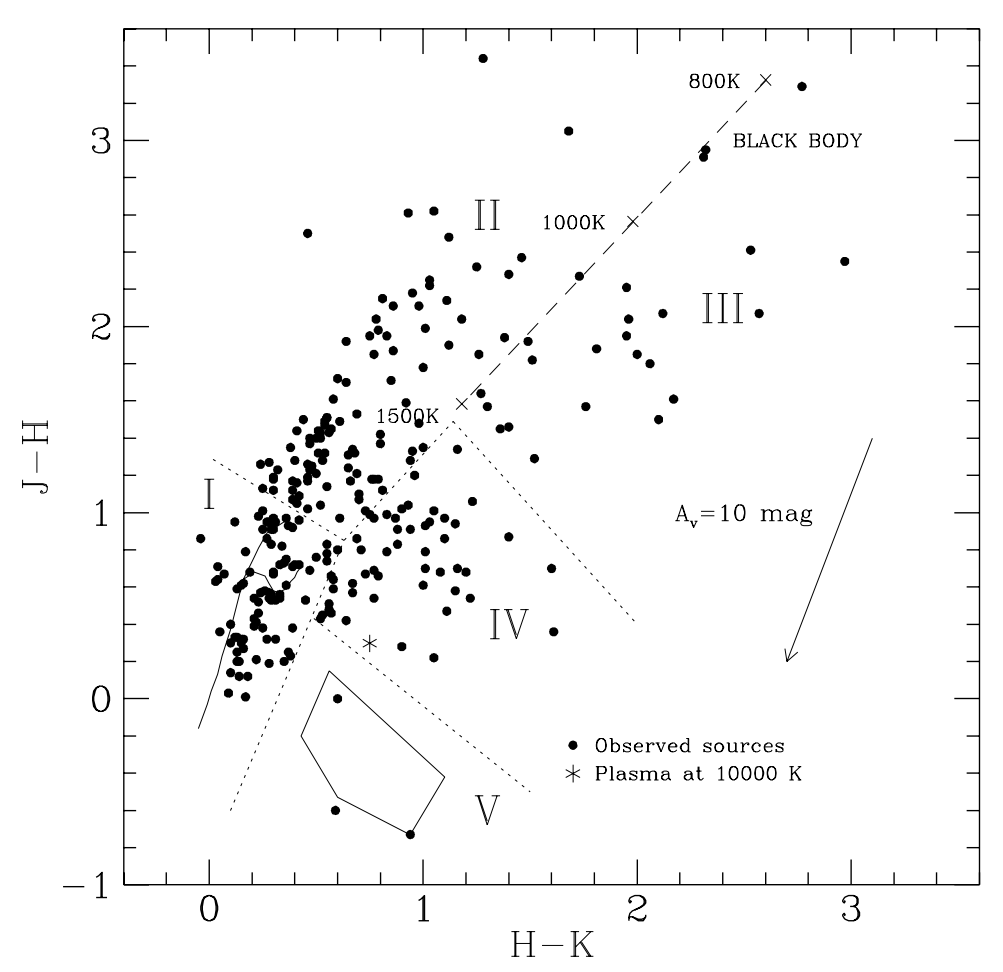

Fig. 3. $J-H$ vs. $H-K$ two-colour diagram where we show the position of the infrared sources observed

sources are found in Region $\mathrm{V}$, where we expect to find well evolved PNe. The three of them have recently been identified as new PNe through optical spectroscopy (García-Lario et al. 1997a), which confirms the validity of this method to detect new PNe.

In contrast, the majority of sources in Fig. 3 are located in Regions I and II of the diagram where basically all kind of objects can be present, as we can see in Table 2, complicating the identification process.

The lack of well evolved PNe among the unidentified objects in our sample can simply be explained as a selection effect. Bright PNe are easily recognized in the optical range through the detection of the many nebular emission lines covering their optical spectra. Those not yet discovered probably belong to the group of very oung and dusty-PNe and, thus, if present in our sample, will probably show unusual near infrared colours, as we will confirm later.

\subsection{Classification of the unidentified IRAS sources}

\subsection{1. $\mathrm{PNe}$}

As we have already shown, only PNe displaying the characteristic excess observed in the $J$ band can be unambiguously recognized as such, based on near infrared data alone. Unfortunately, only 3 objects have been found in Region $\mathrm{V}$ of the near infrared two-colour diagram among the unidentified IRAS sources in our sample. These are SAO 244567, IRAS 18186-0833 and IRAS 17074-1845, and the three of them have been identified as new $\mathrm{PNe}$ through optical spectroscopy.

For the detection of the rest of PNe showing peculiar near infrared colours we need to use additional information obtained in other spectral ranges. The far infrared IRAS colours are not useful in this case, since $\mathrm{PNe}$ are known to be widely distributed throughout the whole selected region in the IRAS two-colour diagram. Fortunately, the ionized gas in their envelopes can easily be detected in the radio continuum and this has been used in a few cases in Table 6 to classify previously unidentified objects as new $\mathrm{PNe}$, once confirmed its nature via optical spectroscopy (van de Steene et al. 1996a,b). In 
Table 3. a) Observed magnitudes

\begin{tabular}{|c|c|c|c|c|c|c|c|}
\hline IRAS name & $J$ & $H$ & $K$ & $L^{\prime}$ & $M$ & Run & Other measurements \\
\hline $00422+6131$ & $12.3 \pm 0.2$ & $12.08 \pm 0.12$ & $11.03 \pm 0.10$ & - & - & $(9)$ & \\
\hline $00470+6130$ & $\geq 12.5$ & $12.07 \pm 0.09$ & $9.85 \pm 0.03$ & - & - & (9) & \\
\hline $02143+5852$ & $10.59 \pm 0.13$ & $9.89 \pm 0.07$ & $8.88 \pm 0.05$ & - & - & (9) & 1 \\
\hline $02395+6244$ & $\geq 12.8$ & $12.15 \pm 0.10$ & $10.29 \pm 0.06$ & - & - & (9) & \\
\hline $02528+4350$ & $9.96 \pm 0.06$ & $9.71 \pm 0.04$ & $9.34 \pm 0.04$ & - & - & (9) & 1 \\
\hline $03578+3134$ & $9.98 \pm 0.07$ & $9.20 \pm 0.03$ & $8.65 \pm 0.03$ & - & - & (9) & 2 \\
\hline $04010+5118$ & $13.4 \pm 0.3$ & $11.46 \pm 0.07$ & $10.08 \pm 0.05$ & - & - & (9) & \\
\hline \multirow[t]{2}{*}{$04101+3103$} & $9.31 \pm 0.02$ & $8.84 \pm 0.02$ & $8.28 \pm 0.02$ & - & - & $(2)$ & 2 \\
\hline & $9.15 \pm 0.04$ & $8.81 \pm 0.03$ & $8.33 \pm 0.03$ & - & - & (9) & \\
\hline $04185+2022$ & $11.69 \pm 0.11$ & $11.08 \pm 0.04$ & $10.72 \pm 0.05$ & - & - & (9) & 2 \\
\hline $04189+2650$ & $10.01 \pm 0.06$ & $8.68 \pm 0.03$ & $7.73 \pm 0.02$ & - & - & (9) & \\
\hline $04296+3429$ & $9.55 \pm 0.07$ & $8.62 \pm 0.06$ & $8.08 \pm 0.06$ & - & - & (8) & 1,3 \\
\hline \multirow[t]{2}{*}{$04302+4425$} & $10.91 \pm 0.17$ & $10.00 \pm 0.06$ & $9.10 \pm 0.06$ & - & - & (8) & 1 \\
\hline & $10.97 \pm 0.15$ & $10.06 \pm 0.13$ & $9.18 \pm 0.09$ & - & - & (9) & \\
\hline $05113+1347$ & $8.88 \pm 0.03$ & $8.35 \pm 0.02$ & $8.11 \pm 0.02$ & $7.1 \pm 0.3$ & - & (9) & 4,5 \\
\hline $05209+2454$ & $6.98 \pm 0.02$ & $6.47 \pm 0.02$ & $5.91 \pm 0.02$ & - & - & (9) & \\
\hline $05238-0626$ & $9.53 \pm 0.04$ & $9.23 \pm 0.03$ & $9.08 \pm 0.03$ & - & - & (9) & 6 \\
\hline $05284+1945$ & - & $\geq 13.7$ & $10.19 \pm 0.06$ & - & - & (8) & \\
\hline \multirow[t]{2}{*}{$05341+0852$} & $9.88 \pm 0.10$ & $9 . \overline{39} \pm 0.06$ & $9.08 \pm 0.05$ & - & - & (8) & 1,6 \\
\hline & $9.94 \pm 0.07$ & $9.41 \pm 0.04$ & $9.10 \pm 0.04$ & - & - & (9) & \\
\hline $05355-0117$ & $8.68 \pm 0.03$ & $7.99 \pm 0.02$ & $7.22 \pm 0.02$ & - & - & (9) & \\
\hline $05471+2351$ & $10.24 \pm 0.11$ & $8.96 \pm 0.03$ & $8.02 \pm 0.02$ & - & - & (9) & 7 \\
\hline \multirow[t]{2}{*}{$05573+3156$} & $\geq 13.0$ & $11.23 \pm 0.09$ & $9.36 \pm 0.03$ & - & - & (3) & \\
\hline & $\geq 12.8$ & $11.27 \pm 0.06$ & $9.46 \pm 0.05$ & - & - & (9) & \\
\hline $05591+1630$ & $8 . \overline{34} \pm 0.03$ & $7.37 \pm 0.02$ & $6.50 \pm 0.02$ & - & - & (9) & 8 \\
\hline \multirow{2}{*}{$06013-1452$} & $9.88 \pm 0.04$ & $9.74 \pm 0.03$ & $9.31 \pm 0.03$ & $8.18 \pm 0.17$ & - & (6) & \\
\hline & $10.01 \pm 0.11$ & $9.58 \pm 0.04$ & $9.06 \pm 0.03$ & - & - & (9) & \\
\hline $06464-1644$ & $11.84 \pm 0.15$ & $10.72 \pm 0.06$ & $9.91 \pm 0.04$ & - & - & (9) & 6 \\
\hline $06499+0145$ & $11.62 \pm 0.04$ & $10.36 \pm 0.03$ & $9.90 \pm 0.02$ & - & - & (2) & \\
\hline \multirow[t]{2}{*}{$06518-1041$} & $\geq 12.7$ & $12.3 \pm 0.2$ & $11.56 \pm 0.11$ & - & - & (3) & 4 \\
\hline & $12.3 \pm 0.3$ & $11.69 \pm 0.11$ & $11.37 \pm 0.09$ & - & - & (9) & \\
\hline $06530-0213$ & $9.66 \pm 0.02$ & $8.94 \pm 0.02$ & $8.52 \pm 0.02$ & - & - & (2) & 9 \\
\hline $06549-2330$ & $9.04 \pm 0.03$ & $8.90 \pm 0.02$ & $8.80 \pm 0.04$ & - & - & (3) & \\
\hline $06556+1623$ & $10.35 \pm 0.09$ & $9.40 \pm 0.03$ & $8.23 \pm 0.03$ & - & - & (9) & 4,7 \\
\hline \multirow[t]{3}{*}{$06562-0337$} & $11.07 \pm 0.13$ & $10.12 \pm 0.08$ & $9.16 \pm 0.05$ & - & - & (3) & 1,10 \\
\hline & $11.28 \pm 0.04$ & $10.48 \pm 0.04$ & $9.71 \pm 0.03$ & $7.80 \pm 0.19$ & - & (6) & \\
\hline & $11.33 \pm 0.10$ & $10.41 \pm 0.04$ & $9.68 \pm 0.03$ & - & - & (9) & \\
\hline $07027-7934$ & $12.11 \pm 0.08$ & $10.26 \pm 0.05$ & $8.26 \pm 0.03$ & $4.85 \pm 0.06$ & $3.82 \pm 0.06$ & (4) & 11,12 \\
\hline $07227-1320$ & $8.89 \pm 0.03$ & $7.92 \pm 0.02$ & $7.62 \pm 0.02$ & $7.06 \pm 0.14$ & - & (4) & \\
\hline
\end{tabular}

addition, optical spectra were also taken for many of the objects located in the IRAS two-colour diagram in the region where no overlap exists with young stellar objects, active galactic nuclei or variable $\mathrm{OH} / \mathrm{IR}$ stars, whenever an optical counterpart in the Palomar or ESO prints was found (García-Lario et al. 1997a). They were the best candidates for being new PNe and, in fact, we identified in this way a few additional $\mathrm{PNe}$ which are also included in Table 6 as new detections. However, our optical spectroscopy revealed that most of the unidentified objects in this region of the diagram were not $\mathrm{PNe}$, but transition objects in the post-AGB stage, some of them showing very bright optical counterparts, as we will see below.

Table 5 shows the distribution of the new $\mathrm{PNe}$ in the near infrared two-colour diagram. As we can see, most of them are associated with colours which are only 
Table 3. b) Observed magnitudes

\begin{tabular}{|c|c|c|c|c|c|c|c|}
\hline IRAS name & $J$ & $H$ & $K$ & $L^{\prime}$ & $M$ & Run & Other measurements \\
\hline $07280-1829$ & $10.18 \pm 0.03$ & $7.97 \pm 0.03$ & $6.02 \pm 0.02$ & - & - & $(2)$ & \\
\hline $07330-2332$ & $12.78 \pm 0.19$ & $11.92 \pm 0.11$ & $10.82 \pm 0.07$ & - & - & (9) & \\
\hline $07399-1435$ & $8.07 \pm 0.02$ & $6.73 \pm 0.02$ & $5.57 \pm 0.02$ & - & - & (9) & 13,14 \\
\hline $07430+1115$ & $9.00 \pm 0.05$ & $8.09 \pm 0.03$ & $7.79 \pm 0.02$ & - & - & (9) & \\
\hline $07582-4059$ & $12.33 \pm 0.08$ & $11.70 \pm 0.07$ & $11.67 \pm 0.08$ & - & - & (6) & 9 \\
\hline $08057-3417$ & $8.82 \pm 0.03$ & $8.28 \pm 0.05$ & $8.07 \pm 0.04$ & $7.68 \pm 0.16$ & - & $(6)$ & \\
\hline $08131-4432$ & $9.63 \pm 0.04$ & $9.18 \pm 0.03$ & $8.65 \pm 0.03$ & $7.3 \pm 0.2$ & - & $(4)$ & \\
\hline $08143-4406$ & $9.25 \pm 0.03$ & $8.71 \pm 0.03$ & $8.43 \pm 0.03$ & $8.1 \pm 0.3$ & - & (4) & \\
\hline $08189+5314$ & $7.44 \pm 0.02$ & $6.77 \pm 0.02$ & $6.70 \pm 0.02$ & - & - & $(9)$ & 15 \\
\hline $08213-3857$ & $9.08 \pm 0.03$ & $8.62 \pm 0.03$ & $8.05 \pm 0.02$ & $6.97 \pm 0.10$ & $6.2 \pm 0.3$ & (4) & \\
\hline $08229-4051$ & $12.38 \pm 0.06$ & $10.96 \pm 0.04$ & $10.16 \pm 0.03$ & - & - & $(6)$ & \\
\hline $08242-3828$ & $7.60 \pm 0.02$ & $6.01 \pm 0.01$ & $5.09 \pm 0.01$ & $3.89 \pm 0.05$ & $3.57 \pm 0.06$ & (4) & \\
\hline $08275-6206$ & $8.33 \pm 0.03$ & $7.42 \pm 0.04$ & $7.17 \pm 0.04$ & $7.00 \pm 0.10$ & - & $(6)$ & \\
\hline $08281-4850$ & $10.68 \pm 0.05$ & $10.12 \pm 0.05$ & $9.79 \pm 0.04$ & - & - & $(6)$ & \\
\hline $08351-4634$ & $12.45 \pm 0.09$ & $11.51 \pm 0.07$ & $11.22 \pm 0.06$ & - & - & $(6)$ & \\
\hline \multirow[t]{2}{*}{$08355-4027$} & $12.43 \pm 0.08$ & $11.48 \pm 0.07$ & $11.21 \pm 0.08$ & - & - & $(4)$ & \\
\hline & $13.34 \pm 0.12$ & $12.81 \pm 0.10$ & $11.65 \pm 0.07$ & - & - & $(6)$ & \\
\hline $08425-5116$ & $13.23 \pm 0.14$ & $11.78 \pm 0.07$ & $10.42 \pm 0.05$ & $7.18 \pm 0.13$ & $6.5 \pm 0.3$ & $(6)$ & 6 \\
\hline $08470-4321$ & $14.2 \pm 0.2$ & $11.85 \pm 0.07$ & $8.88 \pm 0.03$ & $4.07 \pm 0.5$ & $2.63 \pm 0.04$ & (4) & $16,17,18$ \\
\hline $09024-5019$ & $12.68 \pm 0.11$ & $11.25 \pm 0.08$ & $10.69 \pm 0.06$ & - & - & (6) & \\
\hline $09119-5150$ & $13.38 \pm 0.21$ & $12.84 \pm 0.18$ & $11.62 \pm 0.08$ & - & - & (6) & \\
\hline $09362-5413$ & $13.16 \pm 0.16$ & $12.37 \pm 0.11$ & $11.36 \pm 0.07$ & - & - & (6) & \\
\hline \multirow[t]{2}{*}{$09425-6040$} & $6.71 \pm 0.02$ & $5.07 \pm 0.02$ & $3.80 \pm 0.01$ & $2.24 \pm 0.04$ & $1.98 \pm 0.04$ & (4) & 12 \\
\hline & $7.04 \pm 0.02$ & $5.38 \pm 0.02$ & $4.04 \pm 0.02$ & $2.49 \pm 0.04$ & $2.18 \pm 0.06$ & (6) & \\
\hline $10029-5553$ & $12.89 \pm 0.11$ & $11.92 \pm 0.07$ & $10.82 \pm 0.07$ & - & - & (4) & 9 \\
\hline $10115-5640$ & $13.56 \pm 0.18$ & $13.20 \pm 0.14$ & $11.59 \pm 0.07$ & - & - & (6) & \\
\hline $10178-5958$ & $11.13 \pm 0.05$ & $10.26 \pm 0.04$ & $8.86 \pm 0.03$ & $6.29 \pm 0.12$ & $5.4 \pm 0.2$ & (4) & 19 \\
\hline $10197-5750$ & $9.25 \pm 0.03$ & $8.32 \pm 0.02$ & $7.31 \pm 0.02$ & $4.76 \pm 0.06$ & $3.69 \pm 0.06$ & (4) & 14 \\
\hline $10215-5916$ & $4.56 \pm 0.02$ & $3.52 \pm 0.02$ & $3.00 \pm 0.02$ & $2.50 \pm 0.04$ & $2.68 \pm 0.04$ & (4) & 20 \\
\hline $10256-5628$ & $11.08 \pm 0.05$ & $9.90 \pm 0.03$ & $9.14 \pm 0.03$ & $\geq 8.2$ & - & (4) & \\
\hline $10348-6320$ & $13.46 \pm 0.13$ & $12.85 \pm 0.10$ & $12.70 \pm 0.15$ & - & - & (7) & \\
\hline $11065-6026$ & $8.15 \pm 0.03$ & $7.39 \pm 0.02$ & $6.89 \pm 0.02$ & $6.38 \pm 0.12$ & $\geq 5.7$ & (4) & 21 \\
\hline SAO 239162 & $7.12 \pm 0.02$ & $6.58 \pm 0.02$ & $5.81 \pm 0.02$ & $4.36 \pm 0.04$ & $3.89 \pm 0.04$ & (4) & 12 \\
\hline SAO 251457 & $6.40 \pm 0.03$ & $5.83 \pm 0.03$ & $5.16 \pm 0.03$ & $4.12 \pm 0.06$ & $3.79 \pm 0.07$ & (6) & 12,22 \\
\hline $11339-6004$ & $13.15 \pm 0.10$ & $11.92 \pm 0.05$ & $11.60 \pm 0.05$ & - & - & (7) & 9 \\
\hline $11387-6113$ & $9.32 \pm 0.03$ & $8.94 \pm 0.03$ & $8.69 \pm 0.03$ & $\geq 8.2$ & - & (4) & \\
\hline $11415-6541$ & $11.36 \pm 0.05$ & $10.78 \pm 0.03$ & $10.52 \pm 0.04$ & - & - & (7) & \\
\hline \multirow[t]{2}{*}{$11438-6330$} & - & - & $11.64 \pm 0.12$ & $3.75 \pm 0.03$ & $2.11 \pm 0.03$ & (4) & 14,23 \\
\hline & - & $\geq 14.2$ & $7.91 \pm 0.02$ & $1.78 \pm 0.03$ & $0.44 \pm 0.05$ & (6) & \\
\hline
\end{tabular}

unusually observed in well known PNe (see Table 2 for comparison). Some are found in Regions III and IV of the diagram, sometimes extremely reddened, such as IRAS 07027-7934. As we have previously shown, PNe in these regions of the diagram are expected to be very young and dusty. On the other hand, others are also observed in Regions I and II and may be PNe in binary systems, where the emission detected in the near infrared is coming from the photosphere of the companion star. This is true, at least, for IRAS 17395-0841, with a star located at only $2^{\prime \prime}$, which is the main responsible for the near infrared emission observed. However, we have found that many of these new PNe show a faint nebular emission of a very low excitation class and bright central stars with a low effective temperature $(\sim 25000 \mathrm{~K})$ which are completely dominating the emission observed in the near infrared. 
Table 3. c) Observed magnitudes

\begin{tabular}{|c|c|c|c|c|c|c|c|}
\hline IRAS name & $J$ & $H$ & $K$ & $L^{\prime}$ & $M$ & Run & Other measurements \\
\hline $11444-6150$ & $10.96 \pm 0.04$ & $10.01 \pm 0.03$ & $9.70 \pm 0.03$ & - & - & $(7)$ & \\
\hline SAO 223245 & $7.68 \pm 0.05$ & $7.15 \pm 0.04$ & $6.86 \pm 0.03$ & $6.34 \pm 0.06$ & $5.80 \pm 0.16$ & (6) & 24,25 \\
\hline $12158-6443$ & $10.54 \pm 0.04$ & $9.71 \pm 0.03$ & $9.42 \pm 0.03$ & - & - & (7) & \\
\hline SAO 239853 & $8.46 \pm 0.03$ & $8.26 \pm 0.03$ & $8.13 \pm 0.02$ & $\geq 8.0$ & - & (4) & 12,20 \\
\hline \multirow{2}{*}{$12358-6323$} & $12.06 \pm 0.07$ & $10.55 \pm 0.04$ & $10.00 \pm 0.04$ & - & - & (4) & \\
\hline & $13.9 \pm 0.3$ & $12.66 \pm 0.11$ & $11.95 \pm 0.10$ & $6.71 \pm 0.07$ & $4.86 \pm 0.08$ & (6) & 9 \\
\hline $12360-5740$ & $10.31 \pm 0.04$ & $9.92 \pm 0.03$ & $9.71 \pm 0.03$ & - & - & (7) & \\
\hline $13110-6629$ & $8.13 \pm 0.03$ & $7.67 \pm 0.02$ & $7.44 \pm 0.02$ & $7.4 \pm 0.2$ & - & (4) & \\
\hline $13203-5917$ & $9.70 \pm 0.03$ & $9.18 \pm 0.02$ & $8.95 \pm 0.03$ & - & - & (6) & \\
\hline $13245-5036$ & $10.92 \pm 0.12$ & $10.72 \pm 0.03$ & $10.58 \pm 0.04$ & - & - & (6) & \\
\hline $13266-5551$ & $9.96 \pm 0.03$ & $9.84 \pm 0.03$ & $9.70 \pm 0.03$ & $\geq 8.4$ & - & (4) & 12 \\
\hline $13356-6249$ & $8.92 \pm 0.03$ & $7.74 \pm 0.02$ & $6.97 \pm 0.02$ & $6.14 \pm 0.04$ & $5.9 \pm 0.2$ & (4) & \\
\hline \multirow[t]{2}{*}{$13421-6125$} & $12.54 \pm 0.07$ & $11.28 \pm 0.05$ & $11.04 \pm 0.08$ & - & - & (4) & \\
\hline & $13.2 \pm 0.4$ & $11.56 \pm 0.14$ & $11.23 \pm 0.17$ & - & - & (6) & \\
\hline $13427-6531$ & - & - & $13.27 \pm 0.21$ & - & - & (6) & \\
\hline \multirow[t]{2}{*}{$13428-6233$} & $12.49 \pm 0.07$ & $10.57 \pm 0.04$ & $9.08 \pm 0.03$ & $6.64 \pm 0.13$ & $5.32 \pm 0.12$ & $(4)$ & 26 \\
\hline & $12.5 \pm 0.4$ & $10.80 \pm 0.12$ & $9.41 \pm 0.07$ & $7.27 \pm 0.10$ & $5.9 \pm 0.2$ & (6) & \\
\hline $13500-6106$ & $14.0 \pm 0.3$ & $10.56 \pm 0.04$ & $9.28 \pm 0.03$ & - & - & (7) & \\
\hline $14079-6402$ & $11.08 \pm 0.05$ & $9.80 \pm 0.03$ & $9.40 \pm 0.03$ & $8.6 \pm 0.3$ & - & (7) & 27 \\
\hline \multirow{2}{*}{$14104-5819$} & $10.30 \pm 0.05$ & $9.07 \pm 0.03$ & $8.59 \pm 0.03$ & - & - & (4) & \\
\hline & $10.35 \pm 0.04$ & $9.07 \pm 0.03$ & $8.58 \pm 0.03$ & $8.2 \pm 0.3$ & - & $(7)$ & \\
\hline $14122-5947$ & $9.12 \pm 0.05$ & $7.08 \pm 0.05$ & $5.90 \pm 0.04$ & $4.48 \pm 0.04$ & $4.33 \pm 0.07$ & (6) & 9 \\
\hline $14177-5824$ & $9.87 \pm 0.03$ & $8.34 \pm 0.03$ & $7.65 \pm 0.02$ & $7.28 \pm 0.14$ & $\geq 6.2$ & (4) & \\
\hline $14247-6148$ & $\geq 13.8$ & $10.52 \pm 0.04$ & $8.30 \pm 0.03$ & $5.70 \pm 0.05$ & $5.2 \overline{1} \pm 0.13$ & $(7)$ & \\
\hline $14331-6435$ & $9.35 \pm 0.03$ & $9.03 \pm 0.03$ & $8.72 \pm 0.03$ & - & - & (4) & \\
\hline $14562-5637$ & $13.37 \pm 0.12$ & $12.12 \pm 0.08$ & $11.64 \pm 0.09$ & - & - & $(7)$ & \\
\hline $15066-5532$ & $10.47 \pm 0.04$ & $9.30 \pm 0.03$ & $8.64 \pm 0.03$ & $8.1 \pm 0.2$ & - & $(4)$ & \\
\hline \multirow[t]{2}{*}{$15103-5754$} & - & $13.09 \pm 0.12$ & $10.48 \pm 0.07$ & $7.5 \pm 0.2$ & - & (4) & \\
\hline & $14.3 \pm 0.3$ & $12.50 \pm 0.09$ & $10.44 \pm 0.07$ & $7.64 \pm 0.11$ & $6.6 \pm 0.3$ & $(7)$ & \\
\hline $15154-5258$ & $12.01 \pm 0.06$ & $11.15 \pm 0.04$ & $10.88 \pm 0.05$ & - & - & $(6)$ & \\
\hline $15406-4946$ & $8.60 \pm 0.02$ & $7.12 \pm 0.02$ & $6.14 \pm 0.02$ & $4.90 \pm 0.04$ & $4.75 \pm 0.15$ & $(4)$ & \\
\hline $15408-5413$ & $7.37 \pm 0.02$ & $4.32 \pm 0.02$ & $2.64 \pm 0.01$ & $0.76 \pm 0.03$ & $0.37 \pm 0.02$ & $(4)$ & $23,28,29,30$ \\
\hline $15514-5323$ & $12.39 \pm 0.13$ & $11.07 \pm 0.07$ & $10.39 \pm 0.06$ & - & - & $(7)$ & 16 \\
\hline $15553-5230$ & $9.31 \pm 0.05$ & $7.20 \pm 0.05$ & $6.22 \pm 0.04$ & $5.39 \pm 0.05$ & $5.39 \pm 0.14$ & $(6)$ & \\
\hline $16342-3814$ & $9.29 \pm 0.03$ & $8.32 \pm 0.02$ & $7.71 \pm 0.02$ & $6.95 \pm 0.09$ & $\geq 6.4$ & (4) & 12,26 \\
\hline $16552-3050$ & $11.41 \pm 0.13$ & $10.62 \pm 0.04$ & $10.45 \pm 0.06$ & - & - & $(5)$ & 9 \\
\hline $16594-4656$ & $9.95 \pm 0.03$ & $8.98 \pm 0.03$ & $8.21 \pm 0.02$ & $6.9 \pm 0.2$ & $5.66 \pm 0.16$ & (4) & 26 \\
\hline
\end{tabular}

They are also probably very young $\mathrm{PNe}$, very similar to M1-26 and CRL 618, although with not such a strong circumstellar reddening.

Finally, we should also mention that some of the objects not detected in the near infrared above our detection limit turned out to be new PNe when observed in the optical. They are faint PNe but they do not show any indication of being young. This supports the idea that only the brightest PNe and those very young and dusty are easily detectable in the near infrared and can explain why we did not detect a large number of evolved PNe among the unidentified objects in our sample. The selection effect is clear and must be taken into account if we want to derive statistical conclusions.

\subsubsection{Late AGB/Post-AGB stars}

As we can see in Table 5, two thirds of the previously unidentified objects in our sample have been classified either as late-AGB or post-AGB stars. Compared to the 
Table 3. d) Observed magnitudes

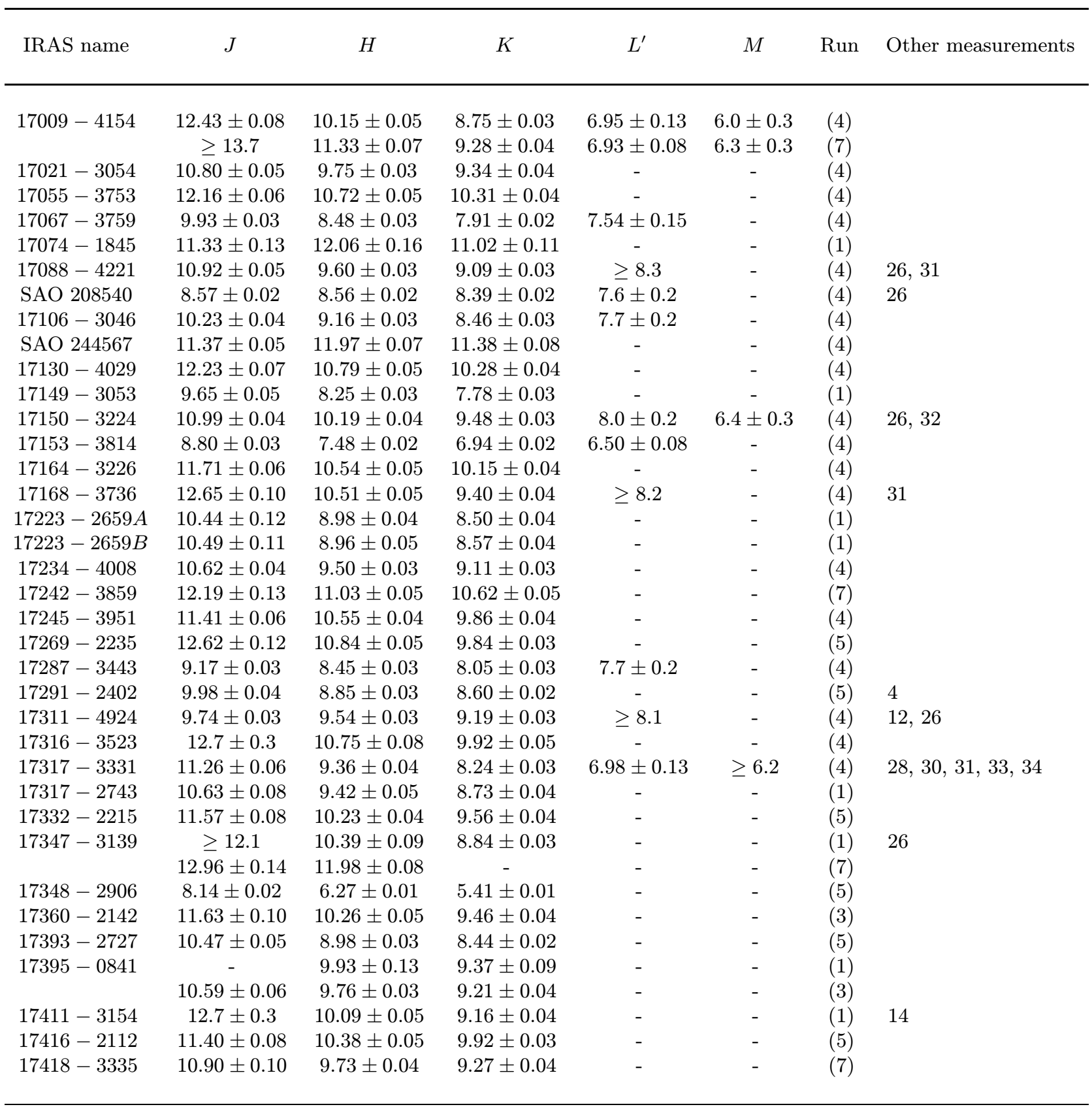

small percentage found among the previously known ones, it seems clear that there also exists a strong selection effect which favours the detection of this kind of objects. This is probably due to the fact that many of these stars can only be identified as such in the far infrared, so that only with the advent of IRAS data it has been possible to recognize stars in this short-lived transition phase which precedes the formation of a $\mathrm{PN}$.
It is important to note that under the group of lateAGB stars in our sample we expect to find only heavily obscured variable $\mathrm{OH} / \mathrm{IR}$ stars, most of them with no optical counterpart in the Palomar or ESO prints. Those with bluer colours or optically bright Mira variables with $\mathrm{OH}$ maser emission, which can also be considered lateAGB stars, are located outside the region of the IRAS twocolour diagram under study, as we can see in Fig. 2. On the 
Table 3. e) Observed magnitudes

\begin{tabular}{|c|c|c|c|c|c|c|c|}
\hline IRAS name & $J$ & $H$ & $K$ & $L^{\prime}$ & $M$ & Run & Other measurements \\
\hline $17423-1755$ & $9.61 \pm 0.05$ & $8.32 \pm 0.02$ & $6.80 \pm 0.02$ & - & - & $(3)$ & \\
\hline $17433-1750$ & $10.22 \pm 0.05$ & $8.95 \pm 0.03$ & $8.67 \pm 0.03$ & - & - & (1) & 9 \\
\hline $17441-2411$ & $11.15 \pm 0.09$ & $9.87 \pm 0.04$ & $9.34 \pm 0.02$ & - & - & (5) & 9,26 \\
\hline SAO 209306 & $7.43 \pm 0.04$ & $7.40 \pm 0.04$ & $7.31 \pm 0.04$ & $7.00 \pm 0.04$ & - & $(6)$ & \\
\hline $17466-3031$ & $11.11 \pm 0.07$ & $8.74 \pm 0.03$ & $7.28 \pm 0.02$ & - & - & $(5)$ & \\
\hline $17479-3032$ & $10.48 \pm 0.05$ & $9.25 \pm 0.03$ & $8.78 \pm 0.03$ & - & - & $(4)$ & \\
\hline $17495-2534$ & $11.8 \pm 0.3$ & $9.30 \pm 0.05$ & $8.84 \pm 0.04$ & - & - & (1) & \\
\hline $17506-2955$ & $10.46 \pm 0.05$ & $9.53 \pm 0.03$ & $9.16 \pm 0.03$ & - & - & $(5)$ & \\
\hline $17540-2753$ & $9.28 \pm 0.03$ & $7.57 \pm 0.03$ & $6.72 \pm 0.03$ & $5.77 \pm 0.09$ & $\geq 7.1$ & $(5)$ & 14 \\
\hline $17542-0603$ & $10.71 \pm 0.15$ & $9.69 \pm 0.07$ & $8.79 \pm 0.07$ & - & - & (1) & \\
\hline $17543-3102$ & $8.84 \pm 0.03$ & $8.16 \pm 0.03$ & $7.97 \pm 0.02$ & $7.8 \pm 0.3$ & - & $(4)$ & \\
\hline $17548-2753 A$ & $12.07 \pm 0.14$ & $10.66 \pm 0.06$ & $10.17 \pm 0.04$ & - & - & $(5)$ & \\
\hline $17548-2753 B$ & $12.25 \pm 0.16$ & $10.95 \pm 0.08$ & $10.68 \pm 0.12$ & - & - & $(5)$ & \\
\hline $17550-2800$ & $10.67 \pm 0.06$ & $9.20 \pm 0.03$ & $8.66 \pm 0.02$ & - & - & $(5)$ & \\
\hline $17550-2120$ & $10.38 \pm 0.06$ & $8.77 \pm 0.02$ & $8.19 \pm 0.02$ & - & - & (5) & \\
\hline $17560-2027$ & $10.19 \pm 0.06$ & $8.34 \pm 0.03$ & $7.57 \pm 0.03$ & - & - & $(3)$ & \\
\hline $17579-3121$ & $8.58 \pm 0.03$ & $8.01 \pm 0.03$ & $7.77 \pm 0.03$ & - & - & (1) & \\
\hline \multirow[t]{2}{*}{$17580-3111$} & $\geq 12.9$ & $11.08 \pm 0.07$ & $9.32 \pm 0.03$ & - & - & $(5)$ & 9 \\
\hline & - & $11.3 \pm 0.3$ & $9.42 \pm 0.08$ & $7.25 \pm 0.08$ & $6.10 \pm 0.24$ & $(6)$ & \\
\hline $17581-2926$ & $11.12 \pm 0.06$ & $10.00 \pm 0.03$ & $9.70 \pm 0.03$ & - & - & (5) & \\
\hline $17582-2619$ & $8.00 \pm 0.02$ & $6.99 \pm 0.02$ & $6.74 \pm 0.02$ & - & - & $(5)$ & \\
\hline $17583-3346$ & $\geq 12.7$ & $13.6 \pm 0.3$ & $10.19 \pm 0.17$ & $5.93 \pm 0.05$ & $5.04 \pm 0.12$ & $(7)$ & \\
\hline $17584-3147$ & $6 . \overline{53} \pm 0.05$ & $5.39 \pm 0.05$ & $4.84 \pm 0.05$ & $4.35 \pm 0.04$ & $4.65 \pm 0.13$ & $(7)$ & \\
\hline $17597-1442$ & $10.33 \pm 0.06$ & $8.93 \pm 0.02$ & $8.41 \pm 0.02$ & - & - & $(3)$ & \\
\hline $18011-2057$ & $11.16 \pm 0.09$ & $9.12 \pm 0.02$ & $8.34 \pm 0.02$ & - & - & $(5)$ & \\
\hline $18019-3121$ & $11.11 \pm 0.10$ & $10.47 \pm 0.09$ & $10.43 \pm 0.11$ & - & - & $(7)$ & \\
\hline $18025-3906$ & $8.60 \pm 0.03$ & $8.06 \pm 0.02$ & $7.73 \pm 0.02$ & $7.49 \pm 0.14$ & $\geq 6.6$ & $(4)$ & 9,12 \\
\hline SAO 85766 & $11.22 \pm 0.08$ & $10.97 \pm 0.07$ & $10.84 \pm 0.08$ & - & - & (3) & 35 \\
\hline $18075-0924$ & $9.25 \pm 0.03$ & $8.29 \pm 0.02$ & $7.87 \pm 0.02$ & - & - & (5) & \\
\hline $18087-1440$ & $11.86 \pm 0.11$ & $9.71 \pm 0.05$ & $8.90 \pm 0.05$ & - & - & (1) & \\
\hline $18095+2704$ & $7.58 \pm 0.01$ & $6.85 \pm 0.01$ & $6.50 \pm 0.01$ & - & - & (5) & $26,35,36,37$ \\
\hline $18096-3230$ & $11.46 \pm 0.08$ & $10.48 \pm 0.11$ & $10.25 \pm 0.10$ & - & - & (7) & \\
\hline $18182-1504$ & $8.45 \pm 0.02$ & $6.23 \pm 0.01$ & $5.20 \pm 0.01$ & - & - & (5) & \\
\hline $18186-0833$ & $11.9 \pm 0.2$ & $11.9 \pm 0.2$ & $11.3 \pm 0.2$ & - & - & (1) & \\
\hline $18216-0156$ & - & $\geq 11.3$ & $9.00 \pm 0.05$ & - & - & (1) & \\
\hline $18229-1127$ & $7.60 \pm 0.03$ & $5 . \overline{61} \pm 0.02$ & $4.60 \pm 0.02$ & - & - & (1) & \\
\hline $18246-1032$ & $10.88 \pm 0.06$ & $9.79 \pm 0.04$ & $9.37 \pm 0.03$ & - & - & (5) & \\
\hline $18252-1016$ & $12.04 \pm 0.17$ & $9.86 \pm 0.05$ & $8.91 \pm 0.03$ & - & - & (5) & \\
\hline
\end{tabular}

other hand, non-variable OH/IR stars, although belonging to the group of post-AGB stars, will be considered as a separate group in the following. As we also see in Table 5, they show near infrared properties which are quite similar to those observed in variable $\mathrm{OH} / \mathrm{IR}$ stars, probably because they have just very recently left the AGB stage and are still heavily obscured by their circumstellar envelopes.

$\mathrm{OH} / \mathrm{IR}$ stars are easily recognized because of the presence of the characteristic double-peaked $\mathrm{OH}$ maser emis- sion at $1612 \mathrm{MHz}$ and the strong silicate absorption feature at $9.8 \mu \mathrm{m}$ in their LRS, when available. However, it is more difficult to determine whether individual objects under this class should be classified either as variable or as non-variable stars. Although, as we will see below their distribution in the IRAS two-colour diagram is not exactly the same, only with a detailed monitoring of the emission observed in the near infrared or in the $\mathrm{OH}$ maser we can unambiguously determine to which of these groups the 
Table 3. f) Observed magnitudes

\begin{tabular}{|c|c|c|c|c|c|c|c|}
\hline IRAS name & $J$ & $H$ & $K$ & $L^{\prime}$ & $M$ & Run & Other measurements \\
\hline $18257-1000$ & $9.03 \pm 0.03$ & $7.08 \pm 0.02$ & $6.33 \pm 0.02$ & - & - & $(1)$ & \\
\hline $18347-0825$ & $\geq 13.0$ & $9.55 \pm 0.05$ & $7.91 \pm 0.02$ & - & - & (5) & \\
\hline $18379-1707$ & $10.76 \pm 0.13$ & $10.55 \pm 0.10$ & $10.33 \pm 0.10$ & - & - & (1) & 12 \\
\hline $18386-1253$ & $9.81 \pm 0.06$ & $9.14 \pm 0.04$ & $8.84 \pm 0.04$ & - & - & (1) & \\
\hline $18420-0512$ & $9.03 \pm 0.03$ & $7.60 \pm 0.02$ & $7.08 \pm 0.03$ & - & - & $(3)$ & \\
\hline $18454+0001$ & $8.86 \pm 0.03$ & $7.16 \pm 0.02$ & $6.52 \pm 0.02$ & - & - & $(5)$ & \\
\hline $18485+0642$ & $11.02 \pm 0.07$ & $9.65 \pm 0.03$ & $9.18 \pm 0.02$ & - & - & (3) & \\
\hline $18514+0019$ & $9.44 \pm 0.07$ & $8.47 \pm 0.04$ & $8.11 \pm 0.04$ & - & - & (1) & \\
\hline $18518+0558$ & $10.82 \pm 0.06$ & $8.71 \pm 0.02$ & $7.85 \pm 0.02$ & - & - & $(5)$ & \\
\hline $18520+0007$ & $11.56 \pm 0.14$ & $9.08 \pm 0.04$ & $7.96 \pm 0.02$ & - & - & (5) & \\
\hline $18576+0341$ & $12.1 \pm 0.3$ & $9.15 \pm 0.03$ & $6.83 \pm 0.02$ & - & - & (1) & \\
\hline $18582+0001$ & $9.22 \pm 0.05$ & $8.50 \pm 0.03$ & $8.17 \pm 0.03$ & - & - & (1) & \\
\hline $19005-0445$ & $10.15 \pm 0.07$ & $8.97 \pm 0.02$ & $8.67 \pm 0.03$ & - & - & (3) & \\
\hline $19071+0857$ & $\geq 13.0$ & $11.90 \pm 0.12$ & $10.62 \pm 0.07$ & - & - & (5) & \\
\hline $19154+0809$ & $12 . \overline{21} \pm 0.13$ & $10.72 \pm 0.04$ & $10.11 \pm 0.04$ & - & - & (3) & \\
\hline $19176+1251$ & $11.49 \pm 0.08$ & $9.57 \pm 0.04$ & $8.93 \pm 0.03$ & - & - & (5) & \\
\hline $19190+1048$ & $12.65 \pm 0.14$ & $10.40 \pm 0.04$ & $9.37 \pm 0.03$ & - & - & (5) & \\
\hline $19193+1804$ & $12.8 \pm 0.2$ & $11.08 \pm 0.07$ & $10.48 \pm 0.06$ & - & - & (5) & \\
\hline $19207+2023$ & $10.35 \pm 0.06$ & $9.43 \pm 0.02$ & $9.04 \pm 0.02$ & - & - & $(5)$ & \\
\hline $19254+1631$ & $11.62 \pm 0.08$ & $9.00 \pm 0.02$ & $7.95 \pm 0.02$ & - & - & (5) & \\
\hline \multirow[t]{2}{*}{$19283+1944$} & - & $\geq 12.9$ & $12.13 \pm 0.17$ & - & - & (5) & \\
\hline & - & - & $11.60 \pm 0.20$ & - & - & (8) & \\
\hline $19306+1407$ & $11.83 \pm 0.13$ & $10.64 \pm 0.05$ & $10.18 \pm 0.05$ & - & - & (5) & \\
\hline $19344+2457$ & $\geq 13.0$ & $12.42 \pm 0.18$ & $9.84 \pm 0.04$ & - & - & (1) & \\
\hline $19356+0754$ & $12.6 \pm 0.2$ & $10.99 \pm 0.08$ & $8.82 \pm 0.03$ & - & - & $(5)$ & \\
\hline $19454+2920$ & $11.90 \pm 0.10$ & $11.16 \pm 0.08$ & $10.61 \pm 0.07$ & - & - & $(5)$ & 35 \\
\hline $19475+3119$ & $7.73 \pm 0.02$ & $7.41 \pm 0.02$ & $7.25 \pm 0.02$ & - & - & $(3)$ & 37 \\
\hline $19477+2401$ & $11.87 \pm 0.15$ & $9.89 \pm 0.04$ & $9.10 \pm 0.03$ & - & - & $(5)$ & 35 \\
\hline $19576+2814$ & $9.94 \pm 0.07$ & $8.43 \pm 0.06$ & $7.88 \pm 0.06$ & - & - & $(8)$ & \\
\hline $19589+4020$ & $11.47 \pm 0.08$ & $10.56 \pm 0.06$ & $10.27 \pm 0.05$ & - & - & $(5)$ & \\
\hline $19590-1249$ & $11.08 \pm 0.12$ & $10.96 \pm 0.14$ & $10.78 \pm 0.10$ & - & - & (1) & \\
\hline $20094+3721$ & $8.06 \pm 0.02$ & $7.31 \pm 0.02$ & $6.95 \pm 0.02$ & - & - & (3) & \\
\hline \multirow[t]{2}{*}{$20144+4656$} & - & $\geq 11.2$ & $10.11 \pm 0.09$ & - & - & (1) & \\
\hline & $12.33 \pm 0.16$ & $11 . \overline{29} \pm 0.08$ & $10.36 \pm 0.05$ & - & - & $(5)$ & \\
\hline $20144+3526$ & $\geq 11.6$ & $11.27 \pm 0.17$ & $9.64 \pm 0.05$ & - & - & (1) & \\
\hline $20244+3509$ & $9 . \overline{50} \pm 0.07$ & $8.26 \pm 0.01$ & $7.61 \pm 0.02$ & - & - & $(3)$ & \\
\hline $20406+2953$ & $11.51 \pm 0.09$ & $9.19 \pm 0.02$ & $7.94 \pm 0.02$ & - & - & $(5)$ & \\
\hline $20461+3853$ & $11.54 \pm 0.11$ & $10.53 \pm 0.05$ & $9.80 \pm 0.04$ & - & - & (9) & \\
\hline
\end{tabular}

source belongs. In the absence of data, the IRAS variability index has been used in Table 6 to classify $\mathrm{OH} / \mathrm{IR}$ stars into one of these two classes. To avoid this problem, whenever it has been possible, the sources showing $\mathrm{OH}$ maser emission in association with a low IRAS variability index were reobserved in the near infrared. In this way, a few objects initially classified as non-variable turned out to be strongly variable. This was the case, for instance, of IRAS 11438-6330, for which we found extraordinary large variations in the near infrared, when observed at two different epochs separated about two years, with a colour index $H-K$ close to 8 magnitudes and an amplitude of more than 3.5 magnitudes in the $K$ band.

With such a red colour, the most heavily obscured variable $\mathrm{OH} / \mathrm{IR}$ stars might not be detectable in the $K$ band, specially if they are observed close to a minimum in their 
Table 3. g) Observed magnitudes

\begin{tabular}{|c|c|c|c|c|c|c|c|}
\hline IRAS name & $J$ & $H$ & $K$ & $L^{\prime}$ & $M$ & Run & Other measurements \\
\hline $20462+3416$ & $10.57 \pm 0.03$ & $10.44 \pm 0.03$ & $10.31 \pm 0.03$ & - & - & (9) & \\
\hline $20470+4458$ & - & $\geq 13.0$ & $10.49 \pm 0.06$ & $6.3 \pm 0.3$ & - & (9) & 1 \\
\hline \multirow[t]{2}{*}{$20490+5934$} & $7.69 \pm 0.05$ & $6 . \overline{70} \pm 0.05$ & $5.95 \pm 0.04$ & - & - & (8) & 4 \\
\hline & $9.84 \pm 0.06$ & $9.36 \pm 0.03$ & $8.82 \pm 0.03$ & - & - & (9) & \\
\hline $20559+6416$ & $9.47 \pm 0.06$ & $8.68 \pm 0.03$ & $7.85 \pm 0.02$ & - & - & (9) & \\
\hline $20572+4919$ & $8.86 \pm 0.03$ & $7.95 \pm 0.02$ & $7.01 \pm 0.02$ & - & - & (9) & 4 \\
\hline $20588+5215$ & $11.20 \pm 0.14$ & $10.10 \pm 0.05$ & $9.40 \pm 0.06$ & - & - & (9) & \\
\hline $21002+4939$ & $9.69 \pm 0.05$ & $8.86 \pm 0.03$ & $7.98 \pm 0.02$ & - & - & (9) & 38 \\
\hline $21289+5815$ & $11.99 \pm 0.22$ & $11.04 \pm 0.09$ & $9.92 \pm 0.05$ & - & - & (9) & \\
\hline $21542+5558$ & $9.95 \pm 0.04$ & $9.27 \pm 0.03$ & $8.97 \pm 0.03$ & - & - & (5) & \\
\hline \multirow[t]{2}{*}{$21546+4721$} & $\geq 13.1$ & $12.34 \pm 0.11$ & $11.41 \pm 0.07$ & - & - & (5) & \\
\hline & $12.9 \pm 0.3$ & $12.29 \pm 0.09$ & $11.29 \pm 0.09$ & - & - & (9) & \\
\hline $22023+5249$ & $11.30 \pm 0.08$ & $11.11 \pm 0.06$ & $10.83 \pm 0.07$ & - & - & (5) & \\
\hline \multirow[t]{3}{*}{$22036+5306$} & $11.82 \pm 0.13$ & $9.75 \pm 0.04$ & $7.63 \pm 0.03$ & - & - & (5) & \\
\hline & $11.6 \pm 0.2$ & $9.55 \pm 0.06$ & $7.51 \pm 0.06$ & - & - & (8) & \\
\hline & $11.55 \pm 0.10$ & $9.60 \pm 0.03$ & $7.50 \pm 0.02$ & $4.70 \pm 0.08$ & - & (9) & \\
\hline \multirow[t]{2}{*}{$22223+4327$} & $8.15 \pm 0.03$ & $7.72 \pm 0.03$ & $7.51 \pm 0.03$ & - & - & $(2)$ & \\
\hline & $7.82 \pm 0.02$ & $7.43 \pm 0.02$ & $7.20 \pm 0.02$ & - & - & (5) & 5 \\
\hline SAO 34504 & $5.55 \pm 0.03$ & $4.91 \pm 0.03$ & $4.67 \pm 0.03$ & - & - & (1) & $1,26,37$ \\
\hline $22331+5809$ & $12.76 \pm 0.14$ & $11.75 \pm 0.09$ & $10.70 \pm 0.05$ & - & - & (5) & \\
\hline $23198-0230$ & $9.19 \pm 0.03$ & $8.20 \pm 0.03$ & $7.37 \pm 0.02$ & - & - & (9) & 39 \\
\hline $23304+6147$ & $8.50 \pm 0.03$ & $7.79 \pm 0.02$ & $7.44 \pm 0.02$ & - & - & (9) & $1,3,4$ \\
\hline $23312+6028$ & $10.13 \pm 0.06$ & $9.81 \pm 0.04$ & $9.54 \pm 0.03$ & - & - & (5) & \\
\hline $23436+6306$ & $11.48 \pm 0.22$ & $10.13 \pm 0.05$ & $9.13 \pm 0.05$ & - & - & (9) & \\
\hline
\end{tabular}

\author{
1. Paper I \\ 2. Kenyon et al. (1990) \\ 3. Hrivnak \& Kwok (1991) \\ 4. Paper II \\ 5. Kwok et al. (1995) \\ 6. Blommaert et al. (1993) \\ 7. Allen (1974) \\ 8. Allen (1973) \\ 9. Hu et al. (1993a) \\ 10. Campbell et al. (1989) \\ 11. Zijlstra et al. (1991) \\ 12. Fouque et al. (1992) \\ 13. Kastner et al. (1992)
}

\author{
14. Lepine et al. (1995) \\ 15. Miroshnichenko et al. (1996) \\ 16. Gaylard \& Whitelock (1988) \\ 17. Persson \& Campbell (1988) \\ 18. Liseau et al. (1992) \\ 19. Allen \& Glass (1975) \\ 20. Hrivnak et al. (1989) \\ 21. Hu et al. (1990) \\ 22. Hu et al. (1989) \\ 23. Gaylard et al. (1989) \\ 24. Elias (1978) \\ 25. Lloyd-Evans (1985) \\ 26. van der Veen et al. (1989)
}

\author{
27. Persi et al. (1987) \\ 28 Le Bertre (1993) \\ 29. Epchtein et al. (1987) \\ 30. Nyman et al. (1993) \\ 31. Epchtein \& Nguyen-Q-Rieu (1982) \\ 32. Hu et al. (1993b) \\ 33. Persi et al. (1990) \\ 34. Jones et al. (1982) \\ 35. Lawrence et al. (1990) \\ 36. Hrivnak et al. (1988) \\ 37. Kastner \& Weintraub (1995) \\ 38. Cohen (1974) \\ 39. Whitelock et al. (1995).
}

light curves. As we can see in Table 5, some objects classified as $\mathrm{OH} / \mathrm{IR}$ stars were not detected in the near infrared in a first visit while, when reobserved at a different epoch, they were succesfuly measured well above the detection limit.

In the near infrared two-colour diagram both variable and non-variable $\mathrm{OH} / \mathrm{IR}$ stars are found strongly concentrated in Region II, as we can see in Table 5.
This region corresponds to objects showing an extremely reddened stellar emission, as expected. In some cases, the near infrared colours are so reddened that the objects fall close to or outside the limits of our plots, as is the case of IRAS $05284+1945$, IRAS 11438-6330, IRAS 14247-6148, IRAS 16437-3140, IRAS 17583-3346 and IRAS $20043+2653$, all them identified as strongly obscured $\mathrm{OH} / \mathrm{IR}$ stars. 
Table 4. IRAS sources not detected in the $K$ band

\begin{tabular}{lccl}
\hline & & & \\
IRAS name & Run & IRAS name & Run \\
\hline & & & \\
$01475-0740$ & $(9)$ & $18011-1847$ & $(3)$ \\
$04117+6402$ & $(9)$ & $18083-2155$ & $(3)$ \\
$04172+4411$ & $(9)$ & $18355-0712$ & $(5)$ \\
$05284+1945^{1}$ & $(9)$ & $18385+1350$ & $(5)$ \\
$05318+2749$ & $(9)$ & $18524+0544$ & $(5)$ \\
$06055-0653$ & $(9)$ & $18533+0523$ & $(5)$ \\
$06499+0145^{2}$ & $(9)$ & $19024+0044$ & $(5)$ \\
$08574-5011$ & $(4)$ & $19182+1806$ & $(5)$ \\
$09024-5019^{3}$ & $(4)$ & $19219+1533$ & $(5)$ \\
$09032-3953$ & $(4)$ & $19480+2504$ & $(5)$ \\
$09370-4826$ & $(6)$ & $20042+3259$ & $(8)$ \\
$10194-5625$ & $(4,7)$ & $20103+3419$ & $(5)$ \\
$11472-7834$ & $(6)$ & $20272+3535$ & $(5)$ \\
$11544-6408$ & $(4)$ & $20404+4527$ & $(5)$ \\
$14562-5637^{4}$ & $(4)$ & $21206+5145$ & $(5)$ \\
$16040-4708$ & $(7)$ & $21388+5622$ & $(5)$ \\
$16114-4504$ & $(4)$ & $21480+5640$ & $(5)$ \\
$17021-3109$ & $(4)$ & $21537+6435$ & $(9)$ \\
$17086-2403$ & $(1)$ & $21554+6204$ & $(9)$ \\
$17448-2131$ & $(1)$ & $22568+6141$ & $(5,9)$ \\
$17521-2938$ & $(5)$ & $23125+5921$ & $(5)$ \\
& & & \\
\hline & & &
\end{tabular}

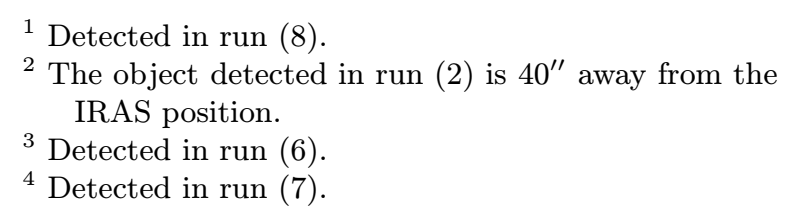

In the far infrared IRAS two-colour diagram, however, variable and non-variable $\mathrm{OH} / \mathrm{IR}$ stars are observed following a different distribution. While objects identified as variable $\mathrm{OH} / \mathrm{IR}$ stars only appear in Region b) of this diagram, as expected, a significant fraction of the IRAS sources classified as non-variable are found well outside the limits of this region, showing a similar distribution to that observed in more evolved post-AGB stars.

Among the IRAS sources classified as post-AGB stars in Table 6 we find not only objects with optically bright counterparts, but also heavily obscured stars still occulted behind their expanding circumstellar shells, showing a low IRAS variability index. Like non-variable $\mathrm{OH} / \mathrm{IR}$ stars, they are also probably in a very early post-AGB stage but, in this case, the $\mathrm{OH}$ maser emission is not detected any more. Most of them are easy to recognize because they are located in the far infrared IRAS two-colour diagram in the region where the only existing overlap is with PNe. Sometimes, however, they are found in regions where a strong overlap exists with other heavily obscured objects, such as compact HII regions or Herbig-Haro objects. In this case, previously unidentified objects have been classified as post-AGB stars and not as young stellar objects only when, after a visual inspection of the Palomar or ESO prints, we found that the IRAS source was not located in the direction of any dark nebula or molecular cloud and, of course, not in association with any known star forming region.

The detection of broad $\mathrm{CO}$ molecular emission lines in some of these post-AGB stars suggests that they may be surrounded by C-rich neutral envelopes. This could be the reason why they were not detected in $\mathrm{OH}$. Some of them must be strongly obscured since they are very bright in the far infrared, with an LRS showing a featureless and very red continuum, but they have not been detected in the $K$ band above our detection limit. This is the case of IRAS $09032-3953$, IRAS $19480+2504$ or IRAS $20028+3910$.

In the near infrared two-colour diagram, heavily obscured post-AGB stars are usually detected in Region II, with an identical distribution to that observed in variable and non-variable $\mathrm{OH} / \mathrm{IR}$ stars, which seems reasonable considering their evolutionary connection. As for the $\mathrm{OH} / \mathrm{IR}$ stars, the near infrared colours observed can be interpreted as stellar emission combined with a moderate circumstellar reddening. With the dilution of the envelope, as a consequence of the expansion of the circumstellar shell, we expect the emission coming from the central star to become dominant in the near infrared. This is the case of the optically bright post-AGB stars found in Region I of the diagram.

The direct connection between non-variable $\mathrm{OH} / \mathrm{IR}$ stars and optically bright post-AGB stars is confirmed by the detection of objects, like IRAS 16559-2957, showing both the characteristic double-peaked $\mathrm{OH}$ maser emission at $1612 \mathrm{MHz}$ and, at the same time, a bright optical counterpart of intermediate spectral type. In this particular case, the detection of a faint $\mathrm{H} \alpha$ emission over an F5 I stellar continuum confirms the right identification of the optical counterpart.

A direct evolutionary connection between heavily obscured post-AGB stars and PNe is also possible, as observed in the case of IRAS 19016-2330. Not detected by us in June 1986, it was observed by van der Veen et al. (1989) in June 1987 with a $K$ magnitude close to our detection limit and very red colours corresponding to our Region III in the near infrared two-colour diagram. Recently, we have detected the faint optical counterpart of this star and we have found that it already shows nebular emission of a very low excitation class over a very red continuum, while the source still show a deep silicate absorption band in the mid-infrared (van der Veen et al. 1989).

The detection of optically bright post-AGB stars with intermediate spectral types is expected in the case of low mass progenitors, since they evolve slow enough to become 
observable again in the optical when the effective temperature of the central is still relatively low. In contrast, high mass progenitor stars would evolve so fast that the central star could reach an effective temperature hot enough to produce the onset of the ionization of the envelope when the circumstellar shell is still optically thick.

Supporting this possibility, a few heavily obscured $\mathrm{OH} / \mathrm{IR}$ stars have been found to show both $\mathrm{OH}$ maser emission and radio continuum emission (Zijlstra et al. 1989). It is tempting to speculate that they may be the result of the rapid evolution of high mass progenitors. However, some of these so-called "OHPN" stars are known to be peculiar. Sometimes, as observed in IRAS 17347-3139, our photometric data indicate that the central star is still strongly variable. In addition, the $\mathrm{OH}$ maser emission observed usually show multiple peaks which have been interpreted as an indication of bipolarity. On the other hand, it is well known that bipolar structures are usually found among type I PNe, which are considered to be the result of the evolution of high mass progenitors. Some of the most heavily obscured new PNe found, like IRAS $07027-7934$ or IRAS 17423-1755, have also been detected in $\mathrm{OH}$, confirming that $\mathrm{OH}$ maser emission and ionization may coexist in some peculiar PNe. At least in the case of IRAS $17423-1755$ we know that the source is strongly bipolar and it shows a very high velocity outflow (Riera et al. 1995).

A considerable number of post-AGB stars with optically bright counterparts are located in Region IV of the diagram. This position cannot be explained only in terms of interstellar or circumstellar reddening and requires the presence of hot dust surrounding the central star. Hot dust is only expected if the mass loss has not completely stopped after the end of the AGB phase. It is well known that some post-AGB stars show sporadic mass loss episodes which can accelerate the transition towards the PN stage. Supporting this interpretation, we have found that most of the post-AGB stars in Region IV of the diagram show $\mathrm{H} \alpha$ emission in their optical spectra (García-Lario et al. 1997a).

A similar situation is observed for many objects in Region I of the diagram identified as post-AGB stars. Some of them are located to the right of the main-sequence and giant stars and, again, this position cannot be explained in terms of interstellar or circumstellar extinction only. The near infrared excess observed is also atributed to hot dust formed as a consequence of recent post-AGB mass loss and this has also been confirmed in many cases through the detection of $\mathrm{H} \alpha$ emission.

\subsubsection{Young stellar objects}

Among the previously unidentified IRAS sources in our sample we have also found a considerable fraction of young stellar objects $(25 \%)$, as expected, most of them concentrated in known star-forming regions, such as the Taurus-
Auriga complex and Orion. This fraction is similar to that observed in the group of well identified IRAS sources. Their identification, however, is not possible based on near infrared data alone, and additional criteria or observations in other spectral ranges have been used.

For this purpose, as already shown, IRAS data can be efficiently used, since we know that T-Tauri and Herbig $\mathrm{Ae} / \mathrm{Be}$ stars are located in a well defined region of the IRAS two-colour diagram. Unfortunately, as we know, in this region we can also find $\mathrm{PNe}$, extremely reddened $\mathrm{OH} / \mathrm{IR}$ stars and galaxies. On the other hand, compact $\mathrm{H}$ II regions and other heavily obscured young stellar objects are also observed only in a very specific region of the IRAS two-colour diagram but, again, some overlap exists, in this case usually with PNe. For the objects located in one of these two overlapping regions, and in the absence of data taken in other spectral ranges, the visual inspection of the Palomar or ESO prints and the search for possible associations with known star forming regions is very useful. In this way, for instance, we have identified IRAS $23312+6028$ as the central star of an extended H II region, clearly visible on the Palomar print.

Of course, the absence of $\mathrm{OH}$ maser emission, the association with a low IRAS variability index or a very low galactic latitude are all criteria which can also be used as additional indicators of a young stellar nature. An abnormal concentration of IRAS sources in a small region of the sky has also been used to identify new star forming regions, specially if the IRAS colours for all them are consistent with a young stellar nature. In addition, sometimes a narrow $\mathrm{CO}$ emission line is observed towards sources embedded in molecular clouds, in contrast with the broad $\mathrm{CO}$ emission line observed in the expanding shells of Crich post-main sequence stars. Moreover, in high density regions, $\mathrm{H}_{2} \mathrm{O}$ maser emission (sometimes also $\mathrm{NH}_{3}$ ), is frequently detected, usually associated to the presence of Herbig-Haro objects.

As we can see in Table 5, most of the previously unidentified IRAS sources now classified as young stellar objects appear concentrated in Regions III and IV of the near infrared two-colour diagram. As expected, the most heavily obscured ones, usually identified as compact H II regions or Herbig-Haro objects, tend to concentrate in Region III of the diagram, although a few of them are also found not too far in Region II. Embedded in their parent molecular clouds, some of them do no show any optical counterpart and the near infrared colours observed are consistent with hot dust emission at temperatures betwen 800 and $1500 \mathrm{~K}$.

In contrast, those located in Region IV of the diagram usually show bright optical counterparts and are identified as T-Tauri or Herbig Ae/Be stars. Many of these stars are known to be surrounded by circumstellar disks which are probably responsible for the strong near infrared excess observed. The few objects identified as T-Tauri or Herbig $\mathrm{Ae} / \mathrm{Be}$ stars found in Region I of the diagram with no or 


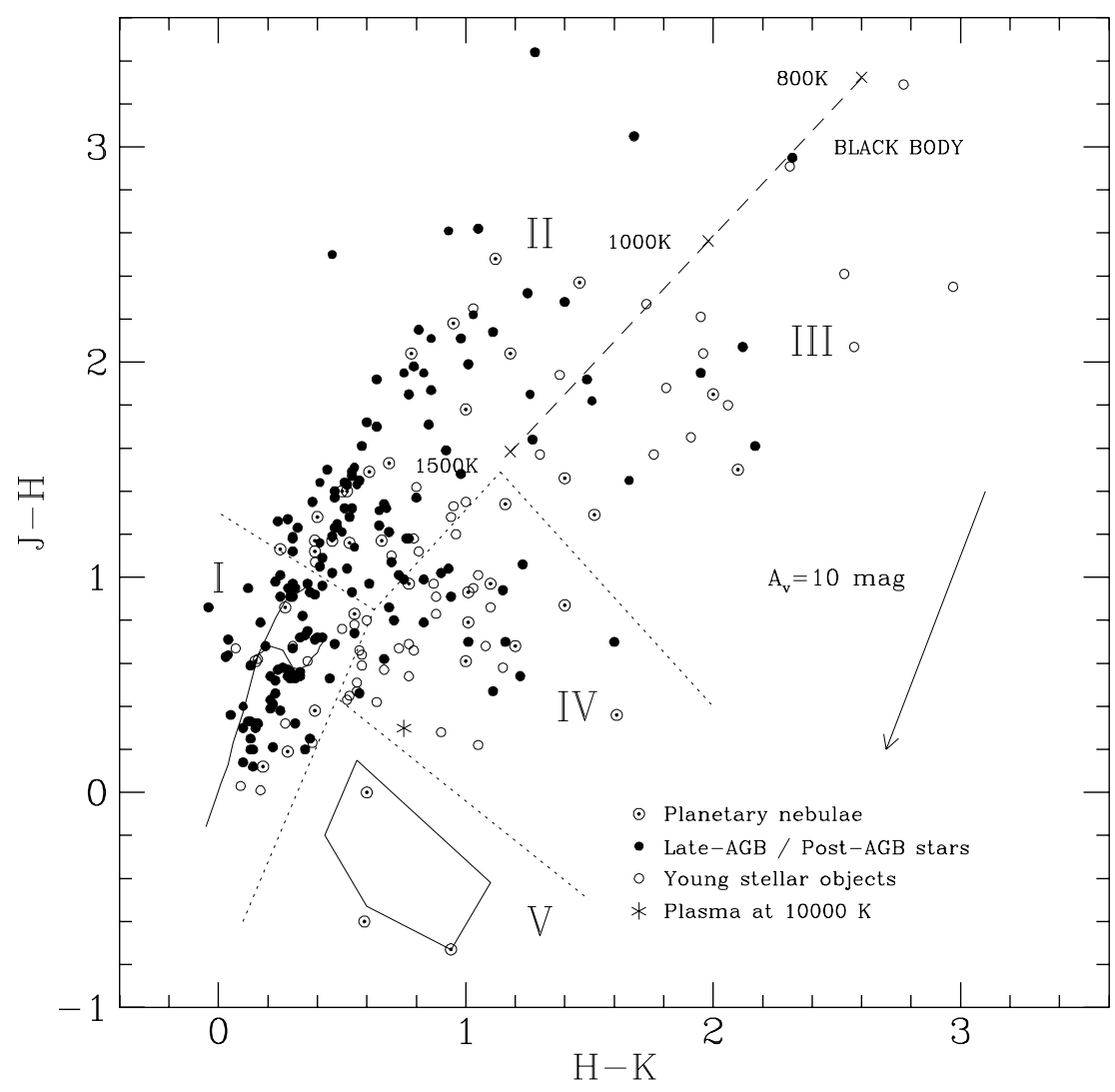

Fig. 4. $J-H$ vs. $H-K$ two colour diagram where we show the position occupied by the observed IRAS sources once they have been classified according to the criteria given in the text

Table 5. Distribution of the various types of objects found among the unidentified IRAS sources in the near infrared two-colour diagram

\begin{tabular}{|c|c|c|c|c|c|c|c|}
\hline Class & Region I & Region II & Region III & Region IV & Region V & Not Detected & Detected (Total) \\
\hline Planetary Nebulae & 8 & 16 & 4 & 9 & 3 & 6 & $40(46)$ \\
\hline Post-AGB & 58 & 28 & 4 & 20 & 0 & 15 & $110(125)$ \\
\hline Non-variable $\mathrm{OH} / \mathrm{IR}$ & 3 & 25 & 3 & 1 & 0 & 4 & $32(36)$ \\
\hline Variable $\mathrm{OH} / \mathrm{IR}$ & 3 & 24 & 1 & 0 & 0 & 3 & $28(31)$ \\
\hline Young Stellar Objects & 11 & 12 & 18 & 26 & 0 & 15 & $67(82)$ \\
\hline Galaxies & 0 & 0 & 0 & 0 & 0 & 2 & $0(2)$ \\
\hline
\end{tabular}




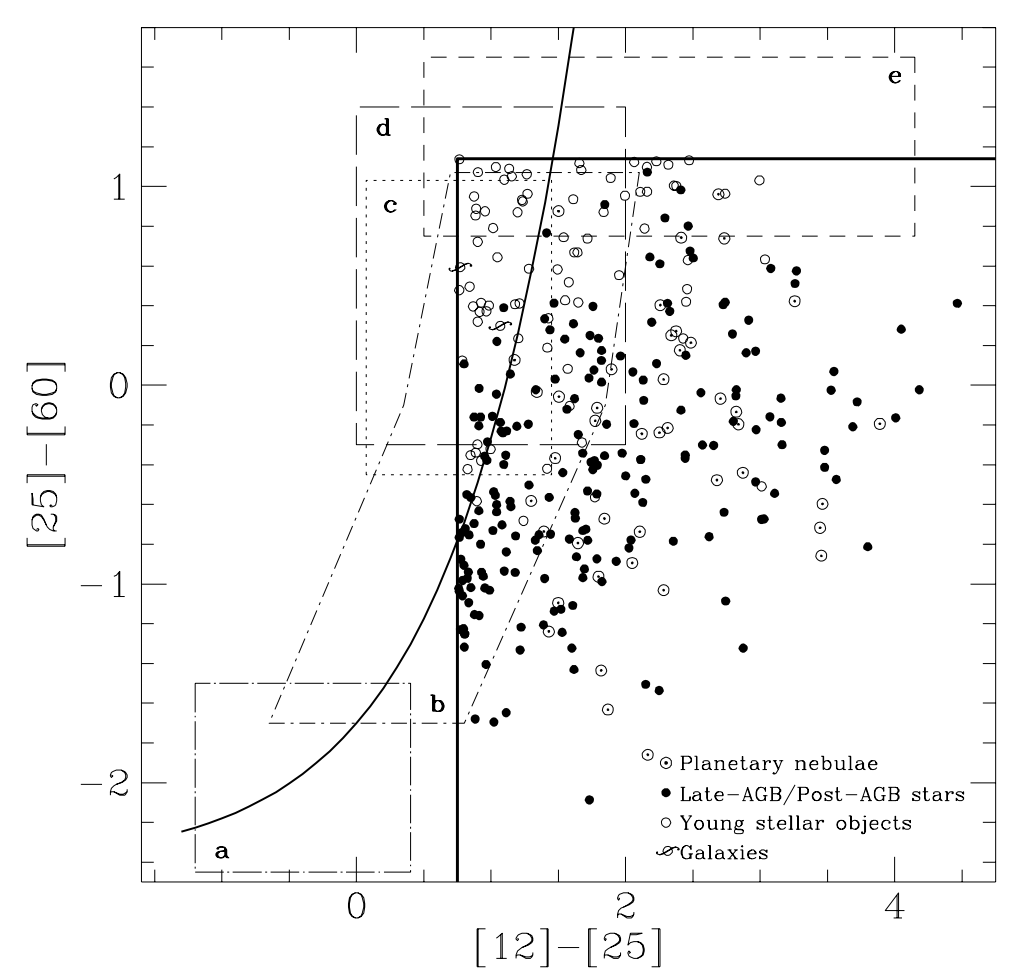

Fig. 5. IRAS two-colour diagram where we show the position of the observed IRAS sources, once they have been classified according to the criteria given in the text

very little near infrared excess may be the result of the observation of these circumstellar disks pole-on.

\subsubsection{Galaxies}

As we know, only the brightest active galactic nuclei are expected to fulfill our selection criteria, and these are usually well known objects in the literature. Thus, it is not surprising that only two objects have been considered as possible new active galactic nuclei among the unidentified IRAS sources in our sample.

One of them, IRAS 01475-0740, has already been confirmed as a new Seyfert galaxy through optical spectroscopy (Pérez et al. 1990). The other one, IRAS $04117+6402$, has tentatively been classified as a possible galaxy because it shows the characteristic colours both in the far infrared and the near infrared and it is located at a relatively high galactic latitude without being associated to any known star forming region. However, a spectroscopic confirmation is still pending.

Although active galactic nuclei appear concentrated in well defined regions of both the far infrared and the near infrared two-colour diagrams, their detection in the near infrared is very difficult, since even the brightest sources are expected to be very faint. In fact, both IRAS $01475-0740$ and IRAS $04117+6402$ were not detected in the $K$ band above our detection limit, suggesting that a few others may be hidden among the rest of sources not detected in the $K$ band listed in Table 4 .

The final classification, including all objects observed in the near infrared so far, is shown in Table 6 . In this Table we give the sequence number of each source in our sample (Col. 1), the IRAS name (Col. 2) together with any other name usually associated to the IRAS source (Col. 3), the IRAS flux at $12 \mu \mathrm{m}$ in Janskys as quoted in Version 2 of the Point Source Catalogue (IRAS Science Team 1988) (Col. 4), the non-colour corrected IRAS colours [12]-[25] (Col. 5) and [25]-[60] (Col. 6) as defined in Sect. 4, the LRS class when available (Col. 7), the IRAS variability index (Col. 8), a code from I to $\mathrm{V}$ indicating the position of the source in the near infrared two-colour diagram or the letters ND if the object was not detected (Col. 9), information about the molecular emission observations carried out in $\mathrm{CO}$ (Cols. 10 and 11) and $\mathrm{OH}$ (Cols. 12 and 13), and our tentative classification (Col. 14). 


\section{Conclusions}

Although, in most cases, based on near infrared data alone, it is not possible to determine the nature of a given source, the combination of our near infrared photometry with the properties observed in the far infrared and a few other additional criteria provides essential information which has been used to determine the nature and evolutionary stage of a sample of unidentified IRAS sources with far infrared colours similar to those observed in well known PNe.

Single near infrared counterparts have been detected in $80 \%$ of the IRAS fields searched. For these positive detections, we have determined the origin of the near infrared emission observed according to whether this is mainly stellar, nebular or due to hot dust (or a combination of them). As shown in Fig. 4, their distribution in the near infrared two-colour diagram is quite different to that shown by the group of well identified objects in our sample plotted in Fig. 1. However, in Fig. 5 we see that there is a very good agreement between the colours observed in the far infrared for the new objects found and those expected for each class, as determined from previous surveys in the literature, which confirms the consistency of the criteria used.

The percentage of young stars found (25\%) is only slightly larger than that previously observed in the sample of well identified IRAS sources, and the new objects found show a very similar distribution in the near infrared twocolour diagram. T-Tauri and Herbig Ae/Be stars usually show stellar-like emission with a moderate near infrared excess which is attributed to the presence of circumstellar disks. Other young stellar objects, such as deeply embedded compact H II regions and Herbig-Haro objects, are strongly obscured and their near infrared colours are consistent with a black-body emission at temperatures betwen 800 and $1500 \mathrm{~K}$.

Only two possible galaxies, both too faint in the near infrared to be detected, were found among the unidentified IRAS sources in our sample. This is explained by the fact that only a small number of bright active galaxies satisfy our selection criteria and most of them were already included in the sample of very well known objects in the literature.

Among the new IRAS sources, we found a very low percentage of $\mathrm{PNe}(13 \%)$, compared to the $49 \%$ observed in our sample of well identified objects. In addition, most of them show peculiar near infrared colours, which are only observed in very young and dusty PNe. The main contribution to the near infrared emission observed in these $\mathrm{PNe}$ comes from their central stars, sometimes affected by a strong circumstellar reddening, and/or from hot dust present in the envelope.

In contrast, we find a very large number of transition objects in the late-AGB or in the post-AGB stage (61\%). They show a wide variety of near infrared colours. Late-
AGB stars are always heavily obscured by their thick circumstellar shells, as expected. Among the stars already in the post-AGB stage we find both, heavily obscured objects (with or without $\mathrm{OH}$ maser emission), and optically bright stars. Some of them show a near infrared excess which is interpreted as the consequence of recent post-AGB mass loss.

Unfortunately, it is not possible just from the relative numbers of post-AGB stars and PNe found to estimate the lifetime of the post-AGB evolutionary phase. First, because it is not clear whether all post-AGB stars become observable $\mathrm{PNe}$ and second, because it is clear that the use of IRAS data produces a strong selection effect which favours the detection of post-AGB stars, which sometimes can only be recognized through the analysis of their far infrared emission. $\mathrm{OH} / \mathrm{IR}$ stars, well evolved $\mathrm{PNe}$ and galaxies are relatively easy to discover in other spectral ranges, because of their strong molecular maser emission, rich emission line optical spectra or the presence of radio continuum emission.

One of the most interesting results obtained is the detection of a relatively large sample of post-AGB stars in a very early stage, still heavily obscured in the optical. Previous surveys have always been biased towards the search for optically bright post-AGB stars with intermediate spectral types, which are probably the result of the evolution of low mass stars which might never become PNe. Among these heavily obscured post-AGB stars we expect to find rapidly evolving massive post-AGB stars which may be the true progenitors of PNe. This possibility is supported by the discovery of a few heavily obscured transition objects already showing the presence of emission lines in their optical spectra and/or radio continuum emission. The detection of their near infrared counterparts is the first step needed for further studies, which are already in progress, including ISO observations in a few cases. This may be crucial to understand the short transition phase which precedes the formation of a PN.

Acknowledgements. This research has made use of the Simbad database, operated at CDS, Strasbourg (France) and it was partially funded through grant PB94-1274 from the Spanish Dirección General de Investigación Científica y Técnica (DGICYT). PGL is the recipient of a Grant from the Spanish Ministerio de Educación y Ciencia.

\section{References}

Allen D.A., 1973, MNRAS 161, 145

Allen D.A., 1974, MNRAS 168, 1

Allen D.A., Glass I.S., 1974, MNRAS 167, 337

Allen D.A., Glass I.S., 1975, MNRAS 170, 579

Allen D.A., Hyland A.R., Caswell J.L., 1980, MNRAS 184, 601

Antonopoulos E., Pottasch S.R., 1987, A\&A 173, 108

Arkhipova V.P., Ikonnikova N.P., 1994, Pis'ma Astron. Zh. 20, 700

Arkhipova V.P., Ikonnikova N.P., Esipov V.F., Noskova R.I., 1996, Pis'ma Astron. Zh. 22, 526 
Arquilla R., Leahy D.A., Kwok S., 1986, MNRAS 220, 125

Arribas S., Martínez Roger C., 1987, A\&AS 70, 303

Becker R.H., White R.L., McLean B.J., Helfand D.J., Zoonematkermani S., 1990, ApJ 358, 485

Bedijn P.J., 1987, A\&A 186, 136

Blommaert J.A.D.L., van der Veen W.E.C.J., Habing H.J., 1993, A\&A 267, 39

Bobrowsky M., 1994, ApJ 426, L47

Bobrowsky M., Zijlstra A.A., Grebel E.K., et al., 1995, ApJ 446, L89

Bouchet P., Manfroid J., Schmider F.X., 1991, A\&AS 91, 409

Brand J., Blitz L., Wouterloot J.G.A., Kerr F.J., 1987, A\&A 68,1

Bronfman L., Nyman L.-A., May J., 1996, A\&AS 115, 81

Bujarrabal V., Bachiller R., 1991, A\&A 242, 247

Bujarrabal V., Alcolea J., Planesas P., 1992, A\&A 257, 701

Bujarrabal V., Fuente A., Omont A., 1994, ApJ 421, L47

Campbell B., Persson S.E., Matthews K., 1989, AJ 98, 643

Carico D.P., Sanders D.B., Soifer B.T., Matthews K., Neugebauer G., 1990, AJ 100, 70

Casoli F. Dupraz C., Gerin M., Combes F., Boulanger F., 1986 A\&A 169, 281

Chapman J.M., 1988, MNRAS 230, 415

Clemens D.P., Barvainis R., 1988, ApJS 68, 257

Cohen M., 1974, MNRAS 169, 257

Cohen M., 1992, AJ 103, 1734

Cohen M., Tielens A.G.G.M., Bregman J., et al., 1989, ApJ 341,246

Crawford T., Marr J., Partridge B., Strauss M.A., 1996, ApJ 460, 225

David P., Le Squeren A.M., Sivagnaman P., 1993, A\&A 277, 453

Davis L.E., Seaquist E.R., Purton C.R., 1979, ApJ 230, 434

Deguchi S., Nakada Y., Forster J.R., 1989, MNRAS 239, 825

de Grijp M.H.K., Miley G.K., Lub J., 1987, A\&AS 70, 95

de Vries C.P., Brand J., Israel F.P., et al., 1984, A\&AS 56, 333

Downes R.A., Keyes C.D., 1988, AJ 96, 777

Eder J., Lewis B.M., Terzian Y., 1988, ApJS 66, 183

Elias J.H., 1978, ApJ 224, 857

Engels D., Lewis B.M., 1996, A\&AS 116, 117

Epchtein N., Nguyen-Q-Rieu, 1982, A\&A 107, 229

Epchtein N., Le Bertre T., Lepine J.R.D., et al., 1987, A\&AS 71,39

Feast M.W., Whitelock P.A., 1987, in "Late stages of stellar evolution", Kwok S. and Pottasch S.R. (eds.). ReidelDordrecht, p. 33

Fekel F.C., Bopp B.W., 1993, ApJ 419, L89

Felli M., Palagi F., Tofani G., 1992, A\&A 255, 293

Fich M., 1993, ApJS 86, 475

Fouque P., Le Bertre T., Epchtein N., Guglielmo F., Kerschbaum F., 1992, A\&AS 93, 151

Frail D.A., Beasley A.J., 1994, A\&A 290, 796

Galt J.A., Kwok S., Frankow J., 1989, AJ 98, 2182

García-Lario P., 1992, Ph. D. Thesis, University of La Laguna, Tenerife, Spain

García-Lario P., Manchado A., Pottasch S.R., Suso J., Olling R., 1990, A\&AS 82, 497 (Paper II)

García-Lario P., Manchado A., Riera A., Mampaso A., Pottasch S.R., 1991, A\&A 249, 223

García-Lario P., Manchado A., Sahu K.C., Pottasch S.R., 1993, A\&A 267, L11
García-Lario P., Manchado A., Parthasarathy M., Pottasch S.R., 1994, A\&A 285, 179

García-Lario P., Sanz Fernández de Córdoba L., Parthasarathy M., Manchado A., Pottasch S.R., 1997a (in preparation)

García-Lario P., Parthasarathy M., de Martino M., et al., 1997b, A\&A (submitted)

Gaylard M.J., Whitelock P.A., 1988, MNRAS 235, 123

Gaylard M.J., West M.E., Whitelock P.A., Cohen R.J., 1989, MNRAS 236, 247

Geballe T.R., van der Veen W.E.C.J., 1990, A\&A 235, L9

Geballe T.R., Tielens A.G.G.M., Kwok S., Hrivnak B.J., 1992, ApJ 387, L89

Gezari D.Y., Schmitz M., Pitts P.S., Mead J.M., 1993, in "Catalog of Infrared Observations. Third Edition", NASA $\mathrm{RP}-1294$

Gómez Y., Moran J.M., Rodríguez L.F., 1990, Rev. Mex. A\&A 20, 55

Gregorio-Hetem J., Lepine J.R.D., Quast G.R., Torres C.A.O., de la Reza R., 1992, AJ 103, 549

Griffith M.R., Wright A.E., Burke B.F., Ekers R.D., 1995, ApJS 97, 347

Guertler J., Kömpe C., Henning T., 1996, A\&A, 305, 878

Gyulbudaghian A.L., Rodríguez L.F., Mendoza-Torres E., 1987, Rev. Mex. A\&A 15, 53

Gyulbudaghian A.L., Rodríguez L.F., Curiel S., 1990, Rev. Mex. A\&A 20, 51

Harris S., Clegg P., Hughes J., 1988, MNRAS 235, 441

Heisler C.A., de Robertis M.M., Nadeau D., 1996, MNRAS 280,579

Henning T., Cesaroni R., Walmsley M., Pfau W., 1992, A\&AS 93,525

Henning T., Chan S.J., Assendorp R., 1996, A\&A 312, 511

Herman J., 1988, A\&AS 74, 133

Herman J., Baud B., Habing H.J., 1985, A\&A 144, 514

Herman J., Habing H.J., 1985, Phys. Reports 124, 257

Hillenbrand L.A., Strom S.E., Vrba F.J., Keene J., 1992, ApJ 398,613

Hrivnak B.J., 1995, ApJ 438, 341

Hrivnak B.J., Kwok S., 1991, ApJ 368, 564

Hrivnak B.J., Kwok S., Volk K.M., 1988, ApJ 331, 832

Hrivnak B.J., Kwok S., Volk K.M., 1989, ApJ 346, 265

Hrivnak B.J., Kwok S., Geballe T.R., 1994, ApJ 420, 783

Hu J.Y., Bibo E.A., 1990, A\&A 234, 435

Hu J.Y., The P.S., de Winter D., 1989, A\&A 208, 213

Hu J.Y., de Winter D., The P.S., Perez M.R., 1990, A\&A 227, L17

Hu J.Y., Slijkhuis S., de Jong T., Jiang B.W., 1993a, A\&AS 100,413

Hu J.Y., Slijkhuis S., Nguyen-Q-Rieu, de Jong T., 1993b, A\&A 273,185

Hu J.Y, te Lintel Hekkert P., Slijkhuis S., Baas R., Wood P.R., 1994, A\&AS 103, 301

IRAS Explanatory Supplement, 1985, Beichman C.A., Neugebauer G., Habing H.J., Clegg P.E., Chester T.J. (eds.). Washington: GPO

IRAS Science Team, 1988, IRAS Point Source Catalogue: Version 2

Jaschek C., Andrillat Y., Jaschek M., 1996, A\&AS 117, 281

Jenness T., Scott P.F., Padman R., 1995, MNRAS 276, 1024

Jones T.J., Hyland A.R., Gatley I., Caswell J.L., 1982, ApJ 253, 208 
Kailey W.F., Lebofsky M.J., 1988, ApJ 326, 653

Kastner J.H., Weintraub D.A., 1995, ApJ 452, 833

Kastner J.H., Weintraub D.A., Zuckerman B., et al., 1992, ApJ 398,552

Kenyon S.J., Hartman L.W., Strom K.M., Strom S.E., 1990, AJ 99, 869

Kistiakowsky V., Helfand D.J., 1995, AJ 110, 2225

Kleinmann S.G., Gillett F.C., Joyce R.R., 1981, ARA\&A 19, 411

Klochkova V.G., 1995, MNRAS 272, 710

Knapp G.R., 1986, ApJ 311, 731

Knapp G.R., Morris, M., 1985, ApJ 292, 640

Knapp G.R., Sutin B.M., Phillips T.G., et al., 1989, ApJ 336, 822

Knapp G.R., Bowers P.F., Young K., Phillips T.G., 1995, ApJ 455, 293

Koornneef J., 1983, A\&AS 51, 489

Kulkarni S.R., Vogel S.N., Wang Z., Wood D.O.S., 1992, Nat 360, 139

Kwok S., Hrivnak B.J., Milone E.F., 1986, ApJ 303, 451

Kwok S., Hrivnak B.J., Boreiko R.T., 1987, ApJ 321, 975

Kwok S., Volk K.V., Hrivnak B.J., 1989, ApJ 345, L51

Kwok S., Hrivnak B.J., Geballe T.R., 1995, ApJ 454, 394

Latter W.B., Maloney P.R., Kelly D.M., et al., 1992, ApJ 389, 347

Lawrence G., Jones T.J., Gehrz R.D., 1990, AJ 99, 1232

Le Bertre T., 1988, The Messenger 51, 24

Le Bertre T., 1993, A\&AS 97, 729

Le Bertre T., Nyman L.-A., 1990, A\&A 233, 477

Lepine J.R.D., Ortiz R., Epchtein N., 1995, A\&A 299, 453

Lewis B., 1992, ApJ 396, 251

Lewis B.M., Eder J, Terzian Y., 1987, AJ 94, 1025

Kwok S., Hrivnak B.J., Milone E.F., 1986, ApJ 303, 451

Kwok S., Hrivnak B.J., Boreiko R.T., 1987, ApJ 321, 975

Kwok S., Volk K.V., Hrivnak B.J., 1989, ApJ 345, L51

Kwok S., Hrivnak B.J., Geballe T.R., 1995, ApJ 454, 394

Latter W.B., Maloney P.R., Kelly D.M., et al., 1992, ApJ 389, 347

Lawrence G., Jones T.J., Gehrz R.D., 1990, AJ 99, 1232

Le Bertre T., 1988, The Messenger 51, 24

Le Bertre T., 1993, A\&AS 97, 729

Le Bertre T., Nyman L.-A., 1990, A\&A 233, 477

Lepine J.R.D., Ortiz R., Epchtein N., 1995, A\&A 299, 453

Lewis B., 1992, ApJ 396, 251

Lewis B., Eder J., Terzian Y., 1990, ApJ 362, 634

Likkel L., 1989, ApJ 344, 350

Likkel L., Morris M., 1988, ApJ 329, 914

Likkel L., Omont A., Morris M., Forveille T., 1987, A\&A 173, L11

Likkel L., Forveille T., Omont A., Morris M., 1991, A\&A 246 , 153

Likkel L., Morris M., Maddalena R.J., 1992, A\&A 256, 581

Lindqvist M. Nyman L.-A., Olofsson H., Winnberg A., 1988, A\&A 205, L15

Liseau R., Lorenzetti D., Nisini B., Spinoglio L., Moneti A., 1992, A\&A 265, 577

Lloyd-Evans T., 1985, MNRAS 217, 493

Loup C., Forveille T., Nyman L.-A., Omont A., 1990, A\&A 227, L29

Luck R.E., Bond H.E., 1989, ApJ 342, 476

MacConnell D.J., 1982, A\&AS 48, 355
Manchado A., Pottasch S.R., García-Lario P., Esteban C., Mampaso A., 1989a, A\&A 214, 139 (Paper I)

Manchado A., García-Lario P., Pottasch S.R., 1989b, Ap\&SS 156,57

Manchado A., García-Lario P., Pottasch S.R., 1989c, A\&A 218, 267

Manchado A., García-Lario P., Sahu K.C., Pottasch S.R., 1990, A\&AS 84, 517

Mauersberger R., Henkel C., Wilson T.L., Olano C.A., 1988, A\&A 206, L34

McCausland R.J.H., Conlon E.S., Dufton P.L., Keenan F.P., 1992, ApJ 394, 298

Menzies J.W., Wolstencroft R.D., 1990, MNRAS 247, 177

Merrill P.W., Burwell C.G., 1950, ApJ 112, 72

Miroshnichenko A.S., Bergner Yu K., Kuratov K.S., 1996, A\&A 312, 521

Morris M., Guilloteau S., Lucas R., Omont A., 1987, ApJ 321, 888

Nyman L.-A., Johansson L.E.B., Booth R.S., 1986, A\&A 160, 352

Nyman L.-A., Booth R.S., Carlstrom U., et al., 1992, A\&AS 93, 121

Nyman L.-A., Hall P.J., Le Bertre T., 1993, A\&A 280, 551

Olnon F.M., Raymond E., 1988, A\&AS 65, 607

Omont A., Loup C., Forveille T., et al., 1993, A\&A 267, 515

Parthasarathy M., 1993, ApJ 414, L109

Parthasarathy M., García-Lario P., Pottasch S.R., et al., 1993, A\&A 267, L19

Parthasarathy M., García-Lario P., de Martino D., et al., 1995, A\&A 300, L25

Peña M., Torres-Peimbert S., 1987, Rev. Mex. A\&A 14, 534

Pérez E., Manchado A., García-Lario P., Pottasch S.R., 1990, A\&A 227, 407

Persi P., Preite-Martinez A., Ferrari-Toniolo M., Spinoglio L., 1987, in "Planetary and proto-planetary nebulae: from IRAS to ISO", Preite-Martinez A. (ed.). Reidel-Dordrecht, p. 221

Persi P., Ferrari-Toniolo M., Busso M., et al., 1988, AJ 95, 1167

Persi P., Ferrari-Toniolo M., Busso M., et al., 1990, AJ 99, 303

Persi P., Palagi F., Felli M., 1994, A\&A 291, 577

Persson S.E., Frogel J.A., 1973, ApJ 182, 503

Persson S.E., Campbell B., 1987, AJ 94, 416

Persson S.E., Campbell B., 1988, AJ 96, 1019

Pollacco D.L., Hill P.W., 1994, MNRAS 267, 692

Pottasch S.R., Bignell C., Zijlstra A.A., 1987, A\&A 177, L49

Pottasch S.R., Bignell C., Olling R., Zijlstra A.A., 1988, A\&A 205, 248

Ratag M., 1991 (private communication)

Ratag M., Pottasch S.R., 1991, A\&AS 91, 481

Ratag M., Pottasch S.R., Zijlstra A.A., Menzies J., 1990, A\&A 233, 181

Reddy B.E., Parthasarathy M., Gonzalez G., Bakker E.J., 1996, ApJ (submitted)

Reipurth B., Wamsteker W., 1983, A\&A 119, 14

Riera A., García-Lario P., Manchado A., Pottasch S.R., Raga A.C., 1995, A\&A 302, 137

Sanders D.B., Soifer B.T., Elias J.H., et al., 1988, ApJ 325, 74

Sevenster M.N., Chapman J.M., Habing H.J., Killeen N.E.B., Lindqvist M., 1997a, A\&AS 122, 79

Sevenster M.N., Chapman J.M., Habing H.J., Killeen N.E.B., Lindqvist M., 1997b, A\&AS (submitted) 
Silva A.M., Azcárate I.N., Poppel W.G.L., Likkel L., 1993, A\&A 275,510

Sivagnaman P., 1989, Ph.D. Thesis, University of Paris VII, France

Skinner C.J., Sylvester R.J., Graham J.R., et al., 1995, ApJ 444,861

Slijkhuis S., Hu J.Y., de Jong T., 1991, A\&A 248, 547

Slysh V.I., Dzura A.M., Val'tts I.E., Gerard E., 1994, A\&AS 106, 87

Strom K.S., Margulis M., Strom S.E., 1989, ApJ 346, L33

Sylvester R.J., Barlow M.J., Skinner C.J., 1994, Ap\&SS 212, 261

Sylvester R.J., Skinner C.J., Barlow M.J., Mannings V., 1996, MNRAS 279, 915

te Lintel Hekkert P., 1991, A\&A 248, 209

te Lintel Hekkert P., Habing H.J., Caswell J.L., Norris R.P., Haynes R.F., 1988, A\&A 202, L19

te Lintel Hekkert P., Versteege-Hansel H.A., Habing H.J., Wiertz M., 1989, A\&AS 78, 399

te Lintel Hekkert P., Caswell J.L., Haynes R.F., Norris R.P., Habing H.J., 1991, A\&AS 90, 327

Torres C.A.O., Quast G., de la Reza R., Gregorio-Hetem J., Lepine J.R.D., 1995, AJ 109, 2146

van der Veen W.E.C.J., Habing H.J., Geballe T.R., 1989, A\&A 226, 108

van der Veen W.E.C.J., Trams N.R., Waters L.B.F.M., 1993, A\&A 269, 231

van de Steene G.C.M., Pottasch S.R., 1993, A\&A 274, 895

van de Steene G.C.M., Jacoby G.H., Pottasch S.R., 1996a, A\&AS 118, 243

van de Steene G.C.M., Sahu K.C., Pottasch S.R., 1996b, A\&AS 120,111

van Langevelde H.J., van der Heiden R., van Schooneveld C., 1990, A\&A 239, 193

van Langevelde H.J., Frail D.A., Cordes J.M., Diamond P.J., 1992, ApJ 396, 686

van Winckel H., Waelkens C., Waters L.B.F.M., 1996, A\&A 306, L37
Volk K., Cohen M., 1989, AJ 98, 931

Volk K., Cohen M., 1990, AJ 100, 485

Volk K., Kwok S., Stencel R.E., Brugel E., 1991, ApJS 77, 607

Volk K., Kwok S., Woodsworth A.W., 1993, ApJ 402, 292

Walker H.J., Wolstencroft R.D., 1988, PASP 100, 1509

Weintraub D.A., Kastner J.H., 1992, BAAS 24, 1141

Whitelock P.A., 1985, MNRAS 213, 59

Whitelock P.A., Menzies J., Feast M., et al., 1994, MNRAS, 267, 711

Whitelock P.A., Menzies J., Feast M., et al., 1995, MNRAS, 276,219

Whiteoak J.B.Z., 1992, A\&A 262, 251

Willner S.P., Becklin E.E., Visvanathan N., 1972, ApJ, 175, 699

Woodsworth A.W., Kwok S., Chan S.J., 1990, A\&A 228, 503

Wouterloot J.G.A., Walmsley C.M., 1986, A\&A 168, 237

Wouterloot J.G.A., Brand J., 1989, A\&AS 80, 149

Wouterloot J.G.A., Brand J., Henkel C., 1988, A\&A 191, 323

Wouterloot J.G.A., Henkel C., Walmsley C.M., 1989, A\&A 215,131

Wouterloot J.G.A., Brand J., Fiegle K., 1993, A\&AS 98, 589

Yun J.L., Clemens D.P., 1994, ApJS 92, 145

Zacs L., Klochkova V.G., Panchuk V.E., 1995, MNRAS 275, 764

Zijlstra A.A., Pottasch S.R., Bignell C., 1990, A\&AS 82, 273

Zijlstra A.A., te Lintel Hekkert P., Pottasch S.R., et al., 1989, A\&A 217, 157

Zijlstra A.A., Gaylard M.J., te Lintel Hekkert P., et al., 1991, A\&A 243, L9

Zoonematkermani S., Helfand D.J., Becker R.H., White R.L., Perley R.A., 1990, ApJS 74, 181

Zuckerman B., Dyck H.M., 1986, ApJ 311, 345

Zuckerman B., Dyck H.M., 1989, A\&A 209, 119

Zuckerman B., Lo K.Y., 1987, A\&A 173, 263

Zuckerman B., Palmer P., Morris M., et al., 1977, ApJ 211, L97

Zuckerman B., Dyck H.M., Claussen M.J., 1986, ApJ 304, 401 